\title{
Langlands duality for representations of quantum groups
}

\author{
Edward Frenkel · David Hernandez
}

Received: 1 November 2008 / Revised: 11 May 2010 / Published online: 12 June 2010 C) The Author(s) 2010. This article is published with open access at Springerlink.com

\begin{abstract}
We establish a correspondence (or duality) between the characters and the crystal bases of finite-dimensional representations of quantum groups associated to Langlands dual semi-simple Lie algebras. This duality may also be stated purely in terms of semi-simple Lie algebras. To explain this duality, we introduce an "interpolating quantum group" depending on two parameters which interpolates between a quantum group and its Langlands dual. We construct examples of its representations, depending on two parameters, which interpolate between representations of two Langlands dual quantum groups.
\end{abstract}

\section{Mathematics Subject Classification (2000) $17 \mathrm{~B} 37$ (17B10, 81R50)}

\section{Introduction}

Let $\mathfrak{g}$ be a simple Lie algebra and ${ }^{L} \mathfrak{g}$ its Langlands dual Lie algebra whose Cartan matrix is the transpose of that of $\mathfrak{g}$. In this paper we establish a duality between finite-dimensional representations of $\mathfrak{g}$ and ${ }^{L} \mathfrak{g}$, as well as the corresponding quantum groups.

\footnotetext{
E. Frenkel was supported in part by DARPA and AFOSR through the grant FA9550-07-1-0543 and by Fondation Sciences mathématiques de Paris.

D. Hernandez was supported partially by ANR through Project "Géométrie et Structures Algébriques Quantiques".
}

E. Frenkel

Department of Mathematics, University of California, Berkeley, CA 94720, USA

D. Hernandez $(\bowtie)$

CNRS, École Normale Supérieure, DMA, 45, rue d’Ulm, 75005 Paris, France

e-mail: David.Hernandez@ens.fr 
Let $I$ be the set of vertices of the Dynkin diagram of $\mathfrak{g}$ and $r_{i}, i \in I$, the corresponding labels. Denote by $r$ the maximal number among the $r_{i}$. This is the lacing number of $\mathfrak{g}$ which is equal to 1 for the simply laced $\mathfrak{g}$, to 2 for $B_{\ell}, C_{\ell}$ and $F_{4}$, and to 3 for $G_{2}$.

Let $L(\lambda)$ be a finite-dimensional irreducible representation of $\mathfrak{g}$ whose highest weight $\lambda$ has the form

$$
\lambda=\sum_{i \in I}\left(1+r-r_{i}\right) m_{i} \omega_{i}, \quad m_{i} \in \mathbb{Z}_{+},
$$

where the $\omega_{i}$ are the fundamental weights of $\mathfrak{g}$. In other words, $\lambda$ is a dominant integral weight which belongs to the sublattice $P^{\prime} \subset P$, where $P$ is the weight lattice of $\mathfrak{g}$, spanned by $\left(1+r-r_{i}\right) \omega_{i}, i \in I$. The character of $L(\lambda)$ has the form

$$
\chi(L(\lambda))=\sum_{\nu \in P} d(\lambda, v) e^{v}, \quad d(\lambda, v) \in \mathbb{Z}_{+} .
$$

Let

$$
\chi^{\prime}(L(\lambda))=\sum_{\nu \in P^{\prime}} d(\lambda, v) e^{v} .
$$

We first prove that, after replacing each

$$
v=\sum_{i \in I}\left(1+r-r_{i}\right) n_{i} \omega_{i} \in P^{\prime}, \quad n_{i} \in \mathbb{Z},
$$

by

$$
v^{\prime}=\sum_{i \in I} n_{i} \check{\omega}_{i}
$$

where the $\check{\omega}_{i}$ are the fundamental weights of ${ }^{L} \mathfrak{g}, \chi^{\prime}(L(\lambda))$ becomes the character of a virtual finite-dimensional representation of ${ }^{L} \mathfrak{g}$, whose highest component is $L\left(\lambda^{\prime}\right)$, the irreducible representation of ${ }^{L} \mathfrak{g}$ with the highest weight

$$
\lambda^{\prime}=\sum_{i \in I} m_{i} \check{\omega}_{i}
$$

where the numbers $m_{i}$ are defined by formula (1). In other words, we have

$$
\chi^{\prime}(L(\lambda))=\chi^{L}\left(L\left(\lambda^{\prime}\right)\right)+\sum_{\check{\mu}<\lambda^{\prime}} m_{\check{\mu}} \chi^{L}(L(\check{\mu})), \quad m_{\check{\mu}} \in \mathbb{Z} .
$$

Then we prove that the multiplicities of weights in the character $\chi^{L}\left(L\left(\lambda^{\prime}\right)\right)$ of $L\left(\lambda^{\prime}\right)$ are less than or equal to those in $\chi^{\prime}(L(\lambda))$. This positivity result means that $\chi^{L}\left(L\left(\lambda^{\prime}\right)\right)$ is "contained as a subcharacter" in $\chi^{\prime}(L(\lambda))$.

Since the categories of finite-dimensional representations of $\mathfrak{g}$ and $U_{q}(\mathfrak{g})$ with generic $q$ are equivalent, we also obtain a duality between finite-dimensional representations of $U_{q}(\mathfrak{g})$ and $U_{q}\left({ }^{L} \mathfrak{g}\right)$. Moreover, we establish the duality not only at the level 
of characters but at the level of crystal bases as well. This leads, in particular, to the following surprising fact: one can construct the crystal basis of the irreducible representation $L\left(\lambda^{\prime}\right)$ of ${ }^{L} \mathfrak{g}$ from the crystal basis of the irreducible representation $L(\lambda)$ of $\mathfrak{g} .{ }^{1}$

In addition, we conjecture that $\chi^{\prime}(L(\lambda))$ is the character of an actual representation of ${ }^{L} \mathfrak{g}$ (that is, $m_{\breve{\mu}} \geq 0$ for all $\check{\mu}$ in formula (3)), and we prove this conjecture for $\mathfrak{g}=B_{2}{ }^{2}$ We observe that the subset of the crystal of $L(\lambda)$ consisting of those elements whose weights are in $P^{\prime}$ does not give us "on the nose" the crystal of this ${ }^{L} \mathfrak{g}$-module. But we conjecture that after applying a certain deformation process (presented in Sect. 6) we do get the right crystal structure on this subset. (We also prove this for $B_{2}$.) Thus, conjecturally, we can reconstruct not only the crystal of $L\left(\lambda^{\prime}\right)$ but the crystal of the whole representation of ${ }^{L} \mathfrak{g}$ whose character is equal to $\chi^{\prime}(L(\lambda))$.

It is natural to ask: why should this duality of characters and crystal bases take place? We suggest the following explanation: there exists an algebra $\mathcal{U}_{q, t}(\mathfrak{g})$ depending on two parameters, $q$ and $t$, whose specialization at $t=1$ gives $\mathcal{U}_{q}(\mathfrak{g})$, and at $q=\epsilon$ (where $\epsilon=1$ if $\mathfrak{g}$ is simply laced and $\epsilon=\exp (\pi i / r), r$ being the lacing number of $\mathfrak{g}$ ) gives $\mathcal{U}_{-t}\left({ }^{L} \mathfrak{g}\right)$. These are the quantum groups without the Serre relations associated to $\mathfrak{g}$ and ${ }^{L} \mathfrak{g}$. We call $\mathcal{U}_{q, t}(\mathfrak{g})$ the interpolating quantum group. (Example 3 in Sect. 5 indicates that it is impossible to include the Serre relations and preserve the interpolating property.) Moreover, we conjecture that any irreducible finite-dimensional representation $L_{q}(\lambda)$ of $U_{q}(\mathfrak{g})$ (equivalently, of $\mathcal{U}_{q}(\mathfrak{g})$ ) with the highest weight of the form (1) may be deformed to a representation $L_{q, t}(\lambda)$ of $\mathcal{U}_{q, t}(\mathfrak{g})$. We also conjecture that the specialization of $L_{q, t}(\lambda)$ at $q=\epsilon$ contains the irreducible representation of $U_{-t}\left({ }^{L} \mathfrak{g}\right)$ with highest weight $\lambda^{\prime}$ given by formula (2) as the highest component. These conjectures are confirmed by various explicit examples presented below as well as our general result on the duality of characters of finite-dimensional representations.

Now we would like to briefly sketch a possible link between our results and the geometric Langlands correspondence (see, e.g., [6] for a general introduction).

One of the key results used in the geometric Langlands correspondence is an isomorphism between the center $Z(\widehat{\mathfrak{g}})$ of the completed enveloping algebra of $\widehat{\mathfrak{g}}$ at the critical level and the classical $\mathcal{W}$-algebra $\mathcal{W}\left({ }^{L} \mathfrak{g}\right)($ see $[4,5]$ as well as [12] for details). This result forms the basis for the local geometric Langlands correspondence $($ see $[7,8])$ as well as for the Beilinson-Drinfeld construction of the global geometric Langlands correspondence [1] (see also [6]). However, this isomorphism is rather mysterious. We know that it exists but we do not fully understand why it should exist.

In order to understand this better, we $q$-deform the picture and consider the center $Z_{q}(\widehat{\mathfrak{g}})$ of the quantum affine algebra $U_{q}(\widehat{\mathfrak{g}})$ at the critical level, which was the starting point of [10]. The center $Z_{q}(\widehat{\mathfrak{g}})$ is in turn related to the Grothendieck ring $\operatorname{Rep} U_{q}(\widehat{\mathfrak{g}})$

\footnotetext{
1 After the first version of this paper appeared on the arXiv, we learned from Nakajima that this result follows from a special case of [18, Theorem 5.1]; see the paragraph before Theorem 2 for more details.

2 After the first version of this paper appeared on the arXiv we were told by Victor Kac that a special case of our duality, going from type $B$ to type $C$, may be explained in the context of representation theory of Lie superalgebras of type $B(0, n)$ as defined in [17]. In fact, the condition on the highest weight $\lambda \in P^{\prime}$ appears in this case in [17, Theorem 8] in the form $a_{n} \in 2 \mathbb{Z}$. It is not clear to us whether one can use Lie superalgebras to interpret our duality for other types. In addition, we have learned from Kevin McGerty that in the meantime he has been able to prove this conjecture for other types (see [23]).
} 
of finite-dimensional representations of $U_{q}(\widehat{\mathfrak{g}})$ (this is because for each finite-dimensional representation $V$ we can construct a generating series of central elements in $Z_{q}(\widehat{\mathfrak{g}})$, using the transfer-matrix construction). Thus, we hope to gain some insight into the isomorphism $Z(\widehat{\mathfrak{g}}) \simeq \mathcal{W}\left({ }^{L} \mathfrak{g}\right)$ by analyzing the connections between $Z_{q}(\widehat{\mathfrak{g}})$, $\operatorname{Rep} U_{q}(\widehat{\mathfrak{g}})$ and the $q$-deformed classical $\mathcal{W}$-algebra.

The idea of [11] was to further deform this picture and introduce a two-parameter (non-commutative) deformation $\mathcal{W}_{q, t}(\mathfrak{g})$. Its specialization $\mathcal{W}_{q, 1}(\mathfrak{g})$ at $t=1$ is the center $Z_{q}(\widehat{\mathfrak{g}})$, so that $\mathcal{W}_{q, t}(\mathfrak{g})$ is a one-parameter deformation of $Z_{q}(\widehat{\mathfrak{g}})$ and a twoparameter deformation of the original center $Z(\widehat{\mathfrak{g}})$. The work [11] was motivated by the hope that analyzing various dualities and limits of $\mathcal{W}_{q, t}(\mathfrak{g})$ we may learn something new about the isomorphism $Z(\widehat{\mathfrak{g}}) \simeq \mathcal{W}\left({ }^{L} \mathfrak{g}\right)$ and hence about the Langlands correspondence.

In particular, it was suggested in [11] that the specialization $\mathcal{W}_{\epsilon, t}(\mathfrak{g})$ at $q=\epsilon$ (with $\epsilon$ defined as above) contains as a subalgebra the center $Z_{t}\left({ }^{L} \widehat{\mathfrak{g}}\right)$ of the quantum affine algebra $U_{t}\left({ }^{L} \widehat{\mathfrak{g}}\right)$ at the critical level (here ${ }^{L} \widehat{\mathfrak{g}}$ denotes the Langlands dual of $\widehat{\mathfrak{g}}$ ). The latter gives rise to the Grothendieck ring of finite-dimensional representations of $U_{t}\left({ }^{L} \widehat{\mathfrak{g}}\right)$ (via the transfer-matrix construction). On the other hand, as we already mentioned above, the specialization $\mathcal{W}_{q, 1}(\mathfrak{g})$ at $t=1$ gives rise to the Grothendieck ring of finite-dimensional representations of $U_{q}(\widehat{\mathfrak{g}})$. Thus, it appears that the $\mathcal{W}$-algebra $\mathcal{W}_{q, t}(\mathfrak{g})$ interpolates between the Grothendieck rings of finite-dimensional representations of quantum affine algebras associated to $\widehat{\mathfrak{g}}$ and ${ }^{L} \widehat{\mathfrak{g}}$. In particular, this suggests that these representations should be related in some way. Examples of such a relation were given in [11], but this phenomenon has largely remained a mystery until now.

How can we explain this relation from the point of view of representation theory? This question served as the motivation for this paper. Before answering it, we considered its finite-dimensional analogue: is there a hidden correspondence, or duality, between finite-dimensional representations of the quantum groups $U_{q}(\mathfrak{g})$ and $U_{q}\left({ }^{L} \mathfrak{g}\right)$-or the simple Lie algebras $\mathfrak{g}$ and ${ }^{L} \mathfrak{g}$, for that matter?

We have given an affirmative answer to this question which we have outlined above. Thus, we have found a hidden duality between objects of the same nature: finitedimensional representations of two Langlands dual Lie algebras. Actually, it is rather surprising to observe the appearance of a Langlands type duality in such an elementary context: that of finite-dimensional representations of simple Lie algebras! We hope that this duality and its affine analogue will give us some clues about the meaning of the geometric Langlands correspondence.

What about the duality for the quantum affine algebras? In our next paper [9] we will propose a precise relation between the $q$-characters of finite-dimensional representations of dual quantum affine algebras $U_{q}(\widehat{\mathfrak{g}})$ and $U_{q}\left({ }^{L} \widehat{\mathfrak{g}}\right)$ with is analogous to the duality of characters of $U_{q}(\mathfrak{g})$ and $U_{q}\left({ }^{L} \mathfrak{g}\right)$ discussed above. We will prove, by using $[14,15]$, that this relation holds for an important class of representations, the Kirillov-Reshetikhin modules. In the affine case we also expect that the duality may be explained by using an affine analogue of the interpolating quantum group.

In the context of our results an interesting problem is to compute explicitly all multiplicities of simple ${ }^{L} \mathfrak{g}$-modules in a given simple $\mathfrak{g}$-module (the numbers $m_{\breve{\mu}}$ in formula (3)), which we call the Langlands duality branching rules. In the course of the proof we have found them explicitly in some cases. 
The paper is organized as follows. In Sect. 2 we establish the duality of characters and crystal bases for a pair of Langlands dual simple Lie algebras. In Sect. 3 we introduce the interpolating quantum group. We then study its representations which we expect to interpolate between representations of $U_{q}(\mathfrak{g})$ and $U_{-t}\left({ }^{L} \mathfrak{g}\right)$. This would explain the duality that we have found in this paper. In Sect. 4 we show how this interpolation works for the finite-dimensional representations of the elementary interpolating quantum groups (those corresponding to Lie algebras of rank one). In Sect. 5 we consider examples of more general interpolating representations. In Sect. 6 we conjecture a stronger duality for characters and crystals and prove it for all simply laced $\mathfrak{g}$ with $r=2$ and for $B_{2}$.

\section{Duality of characters and crystals for simple Lie algebras}

In this section we prove the Langlands duality for characters of finite-dimensional representations of quantum groups associated to simple Lie algebras (or, equivalently, simple Lie algebras themselves). We also prove the duality of the corresponding crystal bases, by using the monomial model [19,24].

Let $\mathfrak{g}$ be a finite-dimensional simple Lie algebra and $U_{q}(\mathfrak{g})$ the corresponding quantum group (see, e.g., [3]). We denote $r=\max _{i \in I}\left(r_{i}\right)$, where $I$ is the set of vertices of the Dynkin diagram of $\mathfrak{g}$ and the $r_{i}$ are the corresponding labels. This is the lacing number of $\mathfrak{g}$ (note that it was denoted by $r^{\vee}$ in [11]).

The Cartan matrix of $\mathfrak{g}$ will be denoted by $C=\left(C_{i, j}\right)_{i, j \in I}$. By definition, the Langlands dual Lie algebra ${ }^{L} \mathfrak{g}$ has the Cartan matrix $C^{t}$, the transpose of the Cartan matrix $C$ of $\mathfrak{g}$.

\subsection{Langlands duality for characters}

Let

$$
P=\sum_{i \in I} \mathbb{Z} \omega_{i}
$$

be the weight lattice of $\mathfrak{g}$ and $P^{+} \subset P$ the set of dominant weights. Consider the sublattice

$$
P^{\prime}=\sum_{i \in I}\left(1+r-r_{i}\right) \mathbb{Z} \omega_{i} \subset P .
$$

Let

$$
P^{L}=\sum_{i \in I} \mathbb{Z} \check{\omega}_{i}
$$

be the weight lattice of ${ }^{L} \mathfrak{g}$. Consider the map $\Pi: P \rightarrow P^{L}$ defined by

$$
\Pi(\lambda)=\sum_{i \in I} \lambda\left(\check{\alpha}_{i}\right)\left(1+r-r_{i}\right)^{-1} \check{\omega}_{i}
$$

if $\lambda \in P^{\prime}$ and $\Pi(\lambda)=0$, otherwise. Clearly, $\Pi$ is surjective. 
In this section we investigate what $\Pi$ does to characters of irreducible representations of $\mathfrak{g}$. For simply laced Lie algebras (that is, $r=1$ ) we have $P^{\prime}=P=P^{L}$ and $\Pi$ is the identity. Hence we focus on the non-simply laced Lie algebras.

Let Rep $\mathfrak{g}$ be the Grothendieck ring of finite-dimensional representations of $\mathfrak{g}$. We have the character homomorphism

$$
\chi: \operatorname{Rep} \mathfrak{g} \rightarrow \mathbb{Z}[P]=\mathbb{Z}\left[y_{i}^{ \pm 1}\right],
$$

where $y_{i}=e^{\omega_{i}}$. It sends an irreducible representation $L(\lambda)$ of $\mathfrak{g}$ with highest weight $\lambda \in P^{+}$to its character, which we will denote by $\chi(\lambda)$.

We will now show that for any representation $V$ of $\mathfrak{g}, \Pi(\chi(V))$ is the character of a virtual representation of ${ }^{L} \mathfrak{g}$, as stated in the following proposition. We denote the character homomorphism for ${ }^{L} \mathfrak{g}$ by $\chi^{L}$.

Proposition 1 For any simple Lie algebra $\mathfrak{g}$ and any $\lambda \in P^{+}, \Pi(\chi(\lambda))$ is in the image of $\chi^{L}$.

This is a direct consequence of the following Lemma. Here we denote by $s_{i}$ (resp. $s_{i}^{L}$ ) the simple reflections of $\mathfrak{g}$ (resp. ${ }^{L} \mathfrak{g}$ ).

Lemma $1 P^{\prime}$ is invariant under the Weyl group action and $\Pi \circ s_{i}=s_{i}^{L} \circ \Pi$ on $P^{\prime}$.

Proof Let $\mu=\prod_{j \in I} y_{j}^{\mu_{j}} \in P^{\prime}$ and $i \in I$.

If $r_{i}=r$, we have $s_{i}(\mu)=\mu y_{i}^{-2 \mu_{i}}\left(\prod_{j \sim i, r_{j}=1} y_{j}^{r \mu_{i}}\right)\left(\prod_{j \sim i, r_{j}=r} y_{i}^{\mu_{i}}\right) \in P^{\prime}$. Moreover $\Pi\left(s_{i}(\mu)\right)=\Pi(\mu) y_{i}^{-2 \mu_{i}}\left(\prod_{j \sim i} y_{j}^{\mu_{i}}\right)=s_{i}^{L}(\Pi(\mu))$.

If $r_{i}=1$, we have $\mu_{i} \in r \mathbb{Z}$ and $s_{i}(\mu)=\mu y_{i}^{-2 \mu_{i}}\left(\prod_{j \sim i} y_{j}^{\mu_{i}}\right) \in P^{\prime}$. Moreover $\Pi\left(s_{i}(\mu)\right)=\Pi(\mu) y_{i}^{-2 \mu_{i}}\left(\prod_{j \sim i, r_{j}=1} y_{j}^{\mu_{i} / r}\right)\left(\prod_{j \sim i, r_{j}=r} y_{j}^{\mu_{i}}\right)=s_{i}^{L}(\Pi(\mu))$.

Remark 1 If $\mathfrak{g}$ is of type $B_{\ell}$ and $\lambda \in P^{+} \cap P^{\prime}$, then all terms in $\chi(\lambda)$ correspond to weights in $P^{\prime}$, and so $\Pi(\chi(\lambda))$ has the same number of monomials as $\chi(\lambda)$.

According to Proposition 1, we have, for $\lambda \in P^{\prime} \cap P^{+}$,

$$
\Pi(\chi(\lambda))=\sum_{\check{\mu} \in P^{L,+}} m_{\check{\mu}} \chi^{L}(\check{\mu}), \quad m_{\check{\mu}} \in \mathbb{Z} .
$$

It is clear from the definition that the maximal $\breve{\mu}$ for which $m_{\breve{\mu}} \neq 0$ is the image of $\lambda$ under $\Pi$. Moreover, in this case $m_{\check{\mu}}=1$. An interesting problem is to compute explicitly all other multiplicities $m_{\breve{\mu}}$, the Langlands duality branching rules.

One of the main results of this section is the following:

Theorem 1 The multiplicities of weights in $\chi^{L}(\Pi(\lambda))$ are less than or equal to those in $\Pi(\chi(\lambda))$.

In other words, $\chi^{L}(\Pi(\lambda))$ can be seen as a "subcharacter" contained in $\Pi(\chi(\lambda))$, that is, $\chi^{L}(\Pi(\lambda)) \preceq \Pi(\chi(\lambda))$ where $\preceq$ is the obvious partial ordering on polynomials. 
Remark 2 In general, the character $\chi(\lambda)$ is given by the Weyl character formula. So one could try to prove the above results by using the Weyl formula. However, it is not clear how to do this: although the Weyl groups of $\mathfrak{g}$ and ${ }^{L} \mathfrak{g}$ are isomorphic, there is no obvious relation for the half-sums of positive roots $\rho$ and $\check{\rho}$.

Before giving the proof, we consider some explicit examples.

Let $\mathfrak{g}=B_{2}$. Then ${ }^{L} \mathfrak{g}=C_{2}$, which is isomorphic to $B_{2}$ but with the switch of the labels of the Dynkin diagram $1 \rightarrow \overline{1}=2,2 \rightarrow \overline{2}=1$. In other words, $\check{\omega}_{i}$ corresponds not to $\omega_{i}$, but to $\omega_{\bar{i}}$.

We have $P^{\prime}=\mathbb{Z} \omega_{1}+2 \mathbb{Z} \omega_{2}$. Here are the simplest examples of action of $\Pi$ on characters of irreducible representations:

$$
\begin{aligned}
\Pi\left(\chi\left(\omega_{1}\right)\right)= & \left(y_{1}+y_{2} y_{1}^{-1}+y_{1} y_{2}^{-1}+y_{1}^{-1}\right)+1 \succeq \chi^{L}\left(\check{\omega}_{1}\right) . \\
\Pi\left(\chi\left(2 \omega_{2}\right)\right)= & \left(y_{2}+y_{2}^{-1} y_{1}^{2}+1+y_{1}^{-2} y_{2}+y_{2}^{-1}\right) \\
& +y_{1}+y_{2} y_{1}^{-1}+1+y_{2}^{-1} y_{1}+y_{1}^{-1} \succeq \chi^{L}\left(\check{\omega}_{2}\right) . \\
\Pi\left(\chi\left(2 \omega_{1}\right)\right)= & \left(y_{1}^{2}+y_{2}+y_{1}^{-2} y_{2}+y_{1}^{2} y_{2}^{-1}+2+y_{1}^{-2} y_{2}+y_{2}^{-2} y_{1}^{2}+y_{2}^{-1}+y_{1}^{-2}\right) \\
& +y_{1}+y_{1}^{-1} y_{2}+y_{2}^{-1} y_{1}+y_{1}^{-1} \succeq \chi^{L}\left(2 \check{\omega}_{1}\right) .
\end{aligned}
$$

Let us look at some examples for $\mathfrak{g}=G_{2}$. In this case ${ }^{L} \mathfrak{g}=G_{2}$, but again with the switch of labels of the Dynkin diagram, as in the case of $B_{2}$. We have $P^{\prime}=\mathbb{Z} \omega_{1}+3 \mathbb{Z} \omega_{3}$. Here are a few examples:

$$
\Pi\left(\chi\left(\omega_{1}\right)\right)=\left(y_{1}+y_{2} y_{1}^{-1}+y_{2}^{-1} y_{1}^{2}+1+y_{1}^{-2} y_{2}+y_{2}^{-1} y_{1}+y_{1}^{-1}\right)+1 \succeq \chi^{L}\left(\check{\omega}_{1}\right) .
$$

$L\left(3 \omega_{2}\right)$ is of dimension 77 . We will not write it out explicitly, but only write

$$
\begin{aligned}
\Pi\left(\chi\left(\omega_{2}\right)\right)= & \left(y_{2}+y_{2}^{-1} y_{1}^{3}+y_{1}+y_{1}^{-1} y_{2}+y_{2}^{2} y_{1}^{-3}+y_{1}^{2} y_{2}^{-1}\right. \\
& \left.+2+y_{1}^{-2} y_{2}+y_{1}^{3} y_{2}^{-2}+y_{1} y_{2}^{-1}+y_{1}^{-1}+y_{2} y_{1}^{-3}+y_{2}^{-1}\right) \\
& +2 y_{1}+2 y_{1}^{-1} y_{2}+2 y_{1}^{2} y_{2}^{-1}+3+2 y_{1}^{-2} y_{2}+2 y_{1} y_{2}^{-1}+2 y_{1}^{-1} \succeq \chi^{L}\left(\check{\omega}_{2}\right) .
\end{aligned}
$$

\subsection{Langlands duality of crystals of irreducible representations}

To prove Theorem 1, we will use the crystal basis theory. It gives us an algorithm to compute character formulas. We will see that the statement of Theorem 1 is actually satisfied at the level of crystal. Before proving this, we state a closely related result describing a duality of crystals of irreducible representations of $U_{q}(\mathfrak{g})$ and $U_{q}\left({ }^{L} \mathfrak{g}\right)$.

Let $\lambda \in P^{\prime} \cap P^{+}$and $\mathcal{B}(\lambda)$ be the corresponding crystal of $L(\lambda)$, with a highest element $u_{\lambda}$ and crystal operators $e_{i}, f_{i}$. We consider the operators

$$
f_{i}^{L}=f_{i}^{1+r-r_{i}}, \quad e_{i}^{L}=e_{i}^{1+r-r_{i}} .
$$

Let $\mathcal{B}^{\prime}(\lambda)$ be the connected component of $u_{\lambda}$ in $\mathcal{B}(\lambda)$ for the operators $f_{i}^{L}, e_{i}^{L}$. Note that the definition of $\mathcal{B}^{\prime}(\lambda)$ depends only on the structure of the $\mathfrak{g}$-crystal of $\mathcal{B}(\lambda)$. 
The weight of the elements of $\mathcal{B}^{\prime}(\lambda)$ are in $P^{\prime}$ and so for $v \in \mathcal{B}^{\prime}(\lambda)$ we can define $\mathrm{wt}^{L}(v)=\Pi(\operatorname{wt}(v))$. Then for any simple Lie algebra $\mathfrak{g}$ (including $G_{2}$ ) we have the following theorem.

After the first version of this paper appeared, we learned from Nakajima that this theorem follows from a special case of [18, Theorem 5.1] (namely, we put $\xi=\operatorname{Id}_{I}$ and $m_{i}=1+r-r_{i}$ in the notation of [18]). Note that [18] discussed examples of embeddings $\mathcal{B}(\lambda) \rightarrow \mathcal{B}(m \lambda)$ and foldings obtained from automorphisms of simply laced Dynkin diagrams, whereas in the present paper we view this in the context of Langlands duality.

Theorem 2 For $\lambda \in P^{\prime} \cap P^{+},\left(\mathcal{B}^{\prime}(\lambda), e_{i}^{L}, f_{i}^{L}, \mathrm{wt}^{L}\right)$ is isomorphic to the ${ }^{L} \mathfrak{g}$-crystal $\mathcal{B}^{L}(\Pi(\lambda))$ of $L(\Pi(\lambda))$.

Thus, by using only the crystal of the $\mathfrak{g}$-module $L(\lambda)$ we have constructed the crystal of the ${ }^{L} \mathfrak{g}$-module $L(\Pi(\lambda))$.

Remark 3 Let us look at $\mathfrak{g}=B_{2}$. If $p$ is even, to the representation $L\left(m \omega_{1}+p \omega_{2}\right)$ of $\mathfrak{g}$ corresponds the representation $L\left(m \omega_{1}+p \omega_{2} / 2\right)$ of ${ }^{L} \mathfrak{g}=C_{2}$. But $C_{2} \simeq B_{2}$. So if in addition $m$ is even, to the representation $L\left(m \omega_{1}+p \omega_{2} / 2\right)$ of ${ }^{L} \mathfrak{g}$ corresponds the representation $L\left(m \omega_{1} / 2+p \omega_{2} / 2\right)$ of ${ }^{L}\left({ }^{L} \mathfrak{g}\right)=\mathfrak{g}$. Thus, we see that this Langlands duality here is not an involution.

Theorem 2 implies Theorem 1 as we have

$$
\Pi\left(\sum_{m^{\prime} \in \mathcal{B}^{\prime}(\lambda)} \operatorname{wt}(m)\right)=\chi^{L}(\Pi(\lambda)) .
$$

\subsection{Reminder: monomial crystals}

Let $C$ be a Cartan matrix of finite type and $s: I \rightarrow\{0,1\}\left(i \mapsto s_{i}\right)$ a map such that $C_{i, j} \leq-1$ implies $s_{i}+s_{j}=1$. Introduce formal variables $Y_{i, l}$, and let $A$ be the set of monomials of the form

$$
m=\prod_{i \in I, l \in \mathbb{Z}} Y_{i, l}^{u_{i, l}(m)}, \quad u_{i, l}(m) \in \mathbb{Z} .
$$

A monomial $m$ is said to be dominant if $\forall j \in I, l \in \mathbb{Z}, u_{j, l}(m) \geq 0$. We set

$$
A_{i, l}=Y_{i, l-1} Y_{i, l+1} \prod_{j \neq i} Y_{j, l}^{C_{j, i}} \in A .
$$

Consider the subgroup $\mathcal{M} \subset A$ defined by

$$
\mathcal{M}=\left\{m \in A \mid u_{i, l}(m)=0 \text { if } l \equiv s_{i}+1 \bmod 2\right\} .
$$


Let us define wt: $A \rightarrow P$ and $\epsilon_{i}, \phi_{i}, p_{i}, q_{i}: A \rightarrow \mathbb{Z} \cup\{\infty\} \cup\{-\infty\}, e_{i}, f_{i}: A \rightarrow$ $A \cup\{0\}$ for $i \in I$ by the formulas (for $m \in A$ )

$$
\begin{aligned}
& \operatorname{wt}(m)=\sum_{i \in I, l \in \mathbb{Z}} u_{i, l}(m) \omega_{i}, \\
& \phi_{i, L}(m)=\sum_{l \leq L} u_{i, l}(m), \quad \phi_{i}(m)=\max \left\{0,\left\{\phi_{i, L}(m) \mid L \in \mathbb{Z}\right\}\right\} \geq 0, \\
& \epsilon_{i, L}(m)=-\sum_{l \geq L} u_{i, l}(m), \quad \epsilon_{i}(m)=\max \left\{0,\left\{\epsilon_{i, L}(m) \mid L \in \mathbb{Z}\right\}\right\} \geq 0, \\
& p_{i}(m)=\max \left\{L \in \mathbb{Z} \mid \epsilon_{i, L}(m)=\epsilon_{i}(m)\right\}, \\
& q_{i}(m)=\min \left\{L \in \mathbb{Z} \mid \phi_{i, L}(m)=\phi_{i}(m)\right\} . \\
& e_{i}(m)= \begin{cases}0 & \text { if } \epsilon_{i}(m)=0, \\
m A_{i, p_{i}(m)-1} & \text { if } \epsilon_{i}(m)>0,\end{cases} \\
& f_{i}(m)= \begin{cases}0 & \text { if } \phi_{i}(m)=0, \\
m A_{i, q_{i}(m)+1}^{-1} & \text { if } \phi_{i}(m)>0 .\end{cases}
\end{aligned}
$$

By $[19,24]\left(\mathcal{M}\right.$, wt $\left., \epsilon_{i}, \phi_{i}, e_{i}, f_{i}\right)$ is a crystal (called the monomial crystal). For $m \in$ $\mathcal{M}$ we denote by $\mathcal{M}(m)$ the subcrystal of $\mathcal{M}$ generated by $m$.

Theorem 3 [19,24] If $m$ is dominant, then the crystal $\mathcal{M}(m)$ is isomorphic to the crystal $\mathcal{B}(\mathrm{wt}(m))$ of $L(\mathrm{wt}(m))$.

In the following we will use the notation $i_{l}^{r}$ for $Y_{i, l}^{r}$.

\subsection{Examples}

We first study examples for Lie algebras of rank 2 and the following representations:

Definition 1 The irreducible representations $L\left(\left(r+1-r_{i}\right) \omega_{i}\right)$ will be called pseudo fundamental representations, and the corresponding highest weights $\left(r+1-r_{i}\right) \omega_{i}$ will be called pseudo fundamental weights.

Note that the pseudo fundamental weights span $P^{\prime}$. By Theorem 2 , the crystals of the pseudo fundamental representations of $\mathfrak{g}$ correspond to the crystals of the fundamental representations of ${ }^{L} \mathfrak{g}$.

Let us start with $B_{2}$. We have the crystal $\mathcal{M}\left(Y_{1,0}\right)$ of the five-dimensional fundamental representation of $\mathcal{U}_{q}\left(B_{2}\right)$ decomposed in $\mathcal{M}^{L}\left(Y_{1,0}\right)$ of the four-dimensional fundamental representation of $\mathcal{U}_{-t}\left(C_{2}\right)$ and to $\mathcal{M}^{L}(1)$ :

$$
\begin{aligned}
& 1_{0} \stackrel{1}{\rightarrow} 1_{2}^{-1} 2_{1}^{2} \stackrel{2}{\rightarrow} 2_{1} 2_{3}^{-1} \stackrel{2}{\rightarrow} 1_{2} 2_{3}^{-2} \stackrel{1}{\rightarrow} 1_{4}^{-1}, \\
& 1_{0} \stackrel{1}{\rightarrow} 1_{2}^{-1} 2_{1} \stackrel{2}{\rightarrow} 1_{2} 2_{3}^{-1} \stackrel{1}{\rightarrow} 1_{4}^{-2} \sqcup\{1\} .
\end{aligned}
$$


Now we have
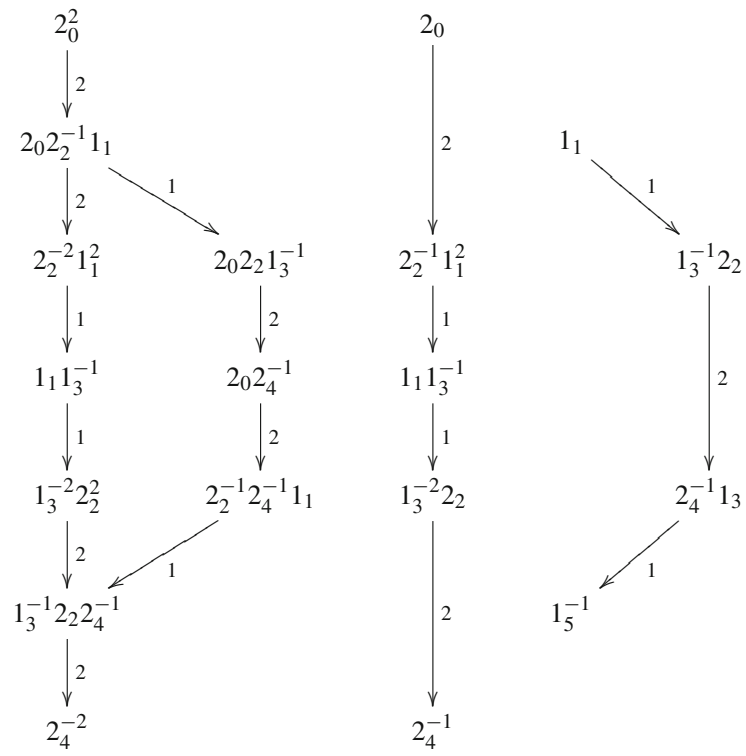

The left crystal is $\mathcal{M}\left(Y_{2,0}^{2}\right)$ corresponding to the ten-dimensional representation $L\left(2 \omega_{2}\right)$ of $\mathcal{U}_{q}\left(B_{2}\right)$. The middle crystal is $\mathcal{M}^{L}\left(Y_{2,0}\right)$ corresponding to the fivedimensional fundamental representation of $\mathcal{U}_{-t}\left(C_{2}\right)$. The two right crystal contain the remaining monomials and are, respectively, isomorphic to $\mathcal{M}^{L}\left(Y_{1,1}\right)$ and $\mathcal{M}^{L}(1)$.

Now we suppose that $\mathfrak{g}$ is of type $G_{2} . \mathcal{M}\left(Y_{1,0}\right)$ has 14 terms $\left\{1_{0}, 1_{2}^{-1} 2_{1}^{3}, 2_{1}^{2} 2_{3}^{-1}, 2_{1} 2_{3}^{-2} 1_{2}, 2_{3}^{-3} 1_{2}^{2}, 2_{1} 2_{3} 1_{4}^{-1}, 1_{2} 1_{4}^{-1}, 2_{1} 2_{5}^{-1}, 1_{4}^{-2} 2_{3}^{3}, 2_{3}^{-1} 2_{5}^{-1} 1_{2}\right.$, $\left.1_{4}^{-1} 2_{3}^{2} 2_{5}^{-1}, 2_{3} 2_{5}^{-2}, 2_{5}^{-3} 1_{4}, 1_{6}^{-1}\right\}$.

The corresponding $\tilde{\mathcal{B}}\left(\omega_{1}\right)$ has terms with two connected components described here. The first component is $\left\{1_{0}, 1_{2}^{-1} 2_{1}^{3}, 2_{3}^{-3} 1_{2}^{2}, 1_{2} 1_{4}^{-1}, 1_{4}^{-2} 2_{3}^{3}\right.$,

$\left.2_{5}^{-3} 1_{4}, 1_{6}^{-1}\right\}$ isomorphic to $\mathcal{B}^{L}\left(\omega_{1}\right)$ and the second component is $\left\{2_{1} 2_{5}^{-1}\right\}$ isomorphic to $\mathcal{B}^{L}(0)$.

$\mathcal{M}\left(Y_{2,0}^{3}\right)$ corresponds to the 77-dimensional representation of $\mathcal{U}_{q}\left(G_{2}\right)$. The corresponding $\tilde{\mathcal{B}}\left(3 \omega_{2}\right)$ has 29 terms with 4 -connected components that we describe. The first component is isomorphic to $\mathcal{B}^{L}\left(\omega_{2}\right)$ :

$\left\{2_{0}^{3}, 2_{2}^{-3} 1_{1}^{3}, 1_{1}^{2} 1_{3}^{-1}, 1_{1} 1_{3}^{-2} 2_{2}^{3}, 1_{3}^{-3} 2_{2}^{6}, 2_{4}^{-3} 1_{1} 1_{3}, 2_{2}^{3} 2_{4}^{-3}, 1_{1} 1_{5}^{-1}, 1_{3}^{-1} 1_{5}^{-1} 2_{2}^{3}, 2_{4}^{-6} 1_{3}^{3}\right.$, $\left.1_{3}^{2} 1_{5}^{-1} 2_{4}^{-3}, 1_{3} 1_{5}^{-2}, 1_{5}^{-3} 2_{4}^{3}, 2_{6}^{-3}\right\}$.

The second component is isomorphic to $\mathcal{B}^{L}\left(\omega_{1}\right):\left\{2_{0} 2_{4}^{-1} 1_{1}\right.$, $\left.1_{3}^{-1} 2_{0} 2_{2}^{3} 2_{4}^{-1}, 2_{0} 2_{4}^{-4} 1_{3}^{2}, 202_{4}^{-1} 1_{3} 1_{5}^{-1}, 1_{5}^{-2} 2_{0} 2_{4}^{2}, 2_{2}^{-1} 2_{6}^{-2} 1_{1}, 2_{2}^{2} 2_{6}^{-2} 1_{3}^{-1}\right\}$.

The third component is isomorphic to $\mathcal{B}^{L}\left(\omega_{1}\right):\left\{2_{0}^{2} 2_{4}^{-2} 1_{3}, 2_{0}^{2} 2_{4} 1_{5}^{-1}\right.$, $\left.2_{2}^{-2} 2_{6}^{-1} 1_{1}^{2}, 2_{2} 2_{6}^{-1} 1_{1} 1_{3}^{-1}, 2_{2}^{4} 2_{6}^{-1} 1_{3}^{-2}, 2_{2} 2_{4}^{-3} 2_{6}^{-1} 1_{3}, 1_{5}^{-1} 2_{2} 2_{6}^{-1}\right\}$.

The fourth component is isomorphic to $\mathcal{B}^{L}(0):\left\{2_{0} 2_{2} 2_{4}^{-1} 2_{6}^{-1}\right\}$. 
Now let us look at the remaining example of Sect. 2.1 for $\mathfrak{g}$ is of type $B_{2}$.
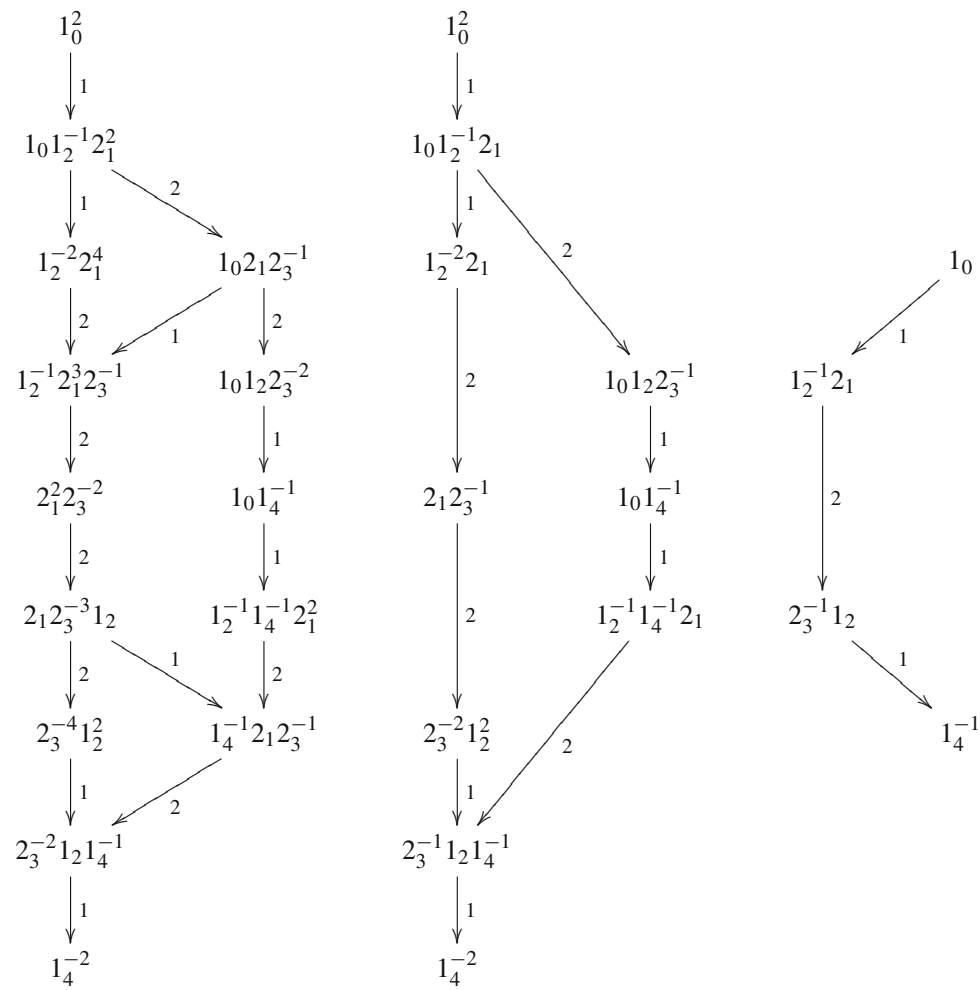

The left crystal is $\mathcal{M}\left(Y_{1,0}^{2}\right)$ corresponding to the 14-dimensional representation $L\left(2 \omega_{1}\right)$ of $\mathcal{U}_{q}\left(B_{2}\right)$. The middle crystal is $\mathcal{M}^{L}\left(Y_{1,0}^{2}\right)$ corresponding to the ten-dimensional representation $L\left(2 \omega_{1}\right)$ of $\mathcal{U}_{-t^{2}}\left(C_{2}\right)$. The right crystal contains the remaining monomials and is isomorphic to $\mathcal{M}^{L}\left(Y_{1,0}\right)$.

\subsection{Proof of Theorem 2}

We consider operators $f_{i}^{L}, e_{i}^{L}$ on $\mathcal{M}$ as defined in formula (5). Let

$$
\begin{aligned}
\mathcal{M}^{\prime} & =\left\{m^{\prime} \in \mathcal{M} \mid \forall i \in I, l \in \mathbb{Z} ; u_{i, l} \in\left(r+1-r_{i}\right) \mathbb{Z}\right\} \\
& =\left\{m^{\prime} \in \mathcal{M} \mid \forall i \in I, l \in \mathbb{Z} ; u_{i, l} \in r \mathbb{Z} \quad \text { if } r_{i}=1\right\} .
\end{aligned}
$$

As $w t\left(\mathcal{M}^{\prime}\right) \subset P^{\prime}$, we can define $\mathrm{wt}^{L}=\Pi \circ \mathrm{w}$ on $\mathcal{M}^{\prime}$.

Lemma 2 Let $i \in I$ such that $r_{i}=1$. Let $m \in \mathcal{M}^{\prime}$ such that $\epsilon_{i}(m)>0$ (resp. $\left.\phi_{i}(m)>0\right)$. Then for $1 \leq q \leq r-1$ we have

(1) $\epsilon_{i}\left(e_{i}^{q}(m)\right)>0\left(\right.$ resp. $\left.\phi_{i}\left(f_{i}^{q}(m)\right)>0\right)$,

(2) $p_{i}\left(e_{i}^{q}(m)\right)=p_{i}(m)\left(\right.$ resp. $\left.q_{i}\left(f_{i}^{q}(m)\right)=q_{i}(m)\right)$,

(3) $e_{i}^{r}(m)=m A_{i, p_{i}(m)-1}^{r}$ (resp. $f_{i}^{r}(m)=m A_{i, q_{i}(m)+1}^{-r}$ ). 
Proof We prove the assertions for $\phi_{i}(m)>0$ (the assertions for $\epsilon_{i}(m)>0$ are proved in the same way).

As $m \in \mathcal{M}^{\prime}$, we have $\phi_{i}(m) \in r \mathbb{Z}$, and so $\phi_{i}(m) \geq r$. So $\phi_{i}\left(f_{i}^{q}(m)\right)=\phi_{i}(m)-q \geq$ 1 and the statement (1) is proved.

We have $f_{i}(m)=m A_{i, q_{i}(m)+1}^{-1}$. We have $\phi_{i, q_{i}(m)}\left(f_{i}(m)\right)=\phi_{i}(m)-1$. For $l \geq q_{i}(m)+2$, we have $\phi_{i, l}\left(f_{i}(m)\right)=\phi_{i, l}(m)-2 \leq \phi_{i}(m)-2$. For $l<q_{i}(m)$, we have $\phi_{i, l}(m) \in r \mathbb{Z}$, so $\phi_{i, l}(m) \leq \phi_{i}(m)-r$ and $\phi_{i, l}\left(f_{i}(m)\right)=\phi_{i, l}(m) \leq \phi_{i}(m)-r$. So $q_{i}\left(f_{i}(m)\right)=q_{i}(m)$ and we have proved the point (2) for $q=1$. If $r=3$ we also have to prove the statement for $q=2$. We have $f_{i}^{2}(m)=m A_{i, q_{i}(m)+1}^{-2}$. We have $\phi_{i, q_{i}(m)}\left(f_{i}(m)^{2}\right)=\phi_{i}(m)-2$. For $l \geq q_{i}(m)+2$, we have $\phi_{i, l}\left(f_{i}^{2}(m)\right)=$ $\phi_{i, l}(m)-4 \leq \phi_{i}(m)-4$. For $l<q_{i}(m)$, we have $\phi_{i, l}(m) \in r \mathbb{Z}$, so $\phi_{i, l}(m) \leq \phi_{i}(m)-r$ and $\phi_{i, l}\left(f_{i}^{2}(m)\right)=\phi_{i, l}(m)<\phi_{i}(m)-2$. So $q_{i}\left(f_{i}(m)\right)=q_{i}(m)$ and we have proved the point (2) for $q=2$.

The last assertion (3) is a direct consequence of the first two assertions.

Let $\Pi: \mathcal{M}^{\prime} \rightarrow \mathcal{M}$ be the map defined by

$$
\Pi(m)=\prod_{i \in I} Y_{i, l}^{u_{i, l}(m)\left(1+r-r_{i}\right)^{-1}} .
$$

Let $\mathcal{M}^{L}$ be the monomial crystal for ${ }^{L} \mathfrak{g}$. Viewed as a set, $\mathcal{M}^{L}$ is equal to $\mathcal{M}$ and so we can view the map $\Pi$ as $\Pi: \mathcal{M}^{\prime} \rightarrow \mathcal{M}^{L}$.

Theorem $4 \mathcal{M}^{\prime} \sqcup\{0\}$ is stable for the operators $f_{i}^{L}, e_{i}^{L}$ which define a structure of ${ }^{L} \mathfrak{g}$-crystal on $\mathcal{M}^{\prime}$. The map $\Pi: \mathcal{M}^{\prime} \rightarrow \mathcal{M}^{L}$ is an isomorphism of ${ }^{L} \mathfrak{g}$-crystals.

Proof The stability for $e_{i}, f_{i}$ when $r_{i}=r$ is clear as the $A_{i, l}^{ \pm} \in \mathcal{M}^{\prime}$. When $r_{i}=1$ it is a consequence of Lemma 2 as the $A_{i, l}^{ \pm r} \in \mathcal{M}^{\prime}$.

To prove that we have a crystal isomorphism, first note that the compatibility of the map with $\epsilon_{i}, \phi_{i}$ is clear. Then for the compatibility with the operators $e_{i}, f_{i}$, it is clear if $r_{i}=r$ and if $r_{i}=1$ it follows from Lemma 2.

Theorem 2 is a direct consequence of Theorem 4. Thus, Theorem 2 is now proved. As discussed above Theorem 1 is also now proved.

Remark 4 The proof given above also implies that Theorems 1 and 2 hold for any symmetrizable Kac-Moody algebra such that $r \leq 3$ (see [16] for their monomial crystal). Here the $r_{i}$ are defined as the set of relatively prime integers such that $r_{i} C_{i, j}=r_{j} C_{j, i}$, and $r$ is the maximal number among the $r_{i}$.

\section{Interpolating quantum groups}

In the previous section we have described a duality between characters and crystal bases of finite-dimensional representations of $U_{q}(\mathfrak{g})$ and $U_{q}\left({ }^{L} \mathfrak{g}\right)$. We would like to explain this duality in the following way: there exists a two-parameter deformation of both of these quantum groups, which we call the "interpolating quantum group". 
Moreover, the dual finite-dimensional representations $U_{q}(\mathfrak{g})$ and $U_{q}\left({ }^{L} \mathfrak{g}\right)$ appear as the result of specialization (of the first and the second parameter, respectively) of a representation of this interpolating quantum group.

In this section we define the interpolating quantum group and in the following two sections we construct their representations which exhibit the desired duality property.

Let again $\mathfrak{g}$ be a finite-dimensional simple Lie algebra and $U_{q}(\mathfrak{g})$ the corresponding quantum group. We denote by $\mathcal{U}_{q}(\mathfrak{g})$ the algebra with the same generators and relations except for the Serre relations. Note that $U_{q}(\mathfrak{g})$ and $\mathcal{U}_{q}(\mathfrak{g})$ have the same categories of finite-dimensional representations.

The interpolating quantum group $\mathcal{U}_{q, t}(\mathfrak{g})$ is an associative algebra depending on two parameters, $q$ and $t$. (Note that this algebra is different from the two-parameter quantum groups considered in [2,25].) We will then establish the following Langlands duality property of these algebras: the specialization with respect to one parameter, $t=1$, gives the quantum group $\mathcal{U}_{q}(\mathfrak{g})$, and the specialization with respect to the other parameter, $q=\epsilon$, where $\epsilon=1$ for simply laced $\mathfrak{g}$ and $\exp (\pi i / r)$ for non-simply laced ones, gives the Langlands dual quantum group $\mathcal{U}_{-t}\left({ }^{L} \mathfrak{g}\right)$.

\subsection{Interpolating simply laced quantum groups $(r=1)$}

Let $\mathfrak{g}$ be a simply laced simple Lie algebra, that is, $r=1$. In this situation the definition of the interpolating quantum group is essentially equivalent to the usual definition of quantum group. In what follows by an "algebra" we will always mean an associative unital algebra over $\mathbb{C}$.

Definition $2 \mathcal{U}_{q, t}(\mathfrak{g})$ is the algebra with the generators $X_{i}^{ \pm}, K_{i}^{ \pm 1}, \widetilde{K}_{i}^{ \pm 1}$ and relations

$$
\begin{aligned}
K_{i} X_{j}^{ \pm} & =q^{ \pm C_{i, j}} X_{j}^{ \pm} K_{i}, \widetilde{K_{i}} X_{j}^{ \pm}=t^{ \pm C_{i, j}} X_{j}^{ \pm} \widetilde{K}_{i}, \\
{\left[X_{i}, X_{j}^{-}\right] } & =\delta_{i, j} \frac{K_{i} \widetilde{K}_{i}-\left(K_{i} \widetilde{K}_{i}\right)^{-1}}{q t-(q t)^{-1}} .
\end{aligned}
$$

Note that

$$
\mathcal{U}_{q, t}(\mathfrak{g}) \supset\left\langle\left(K_{i} \tilde{K}_{i}\right)^{ \pm 1}, X_{i}^{ \pm}\right\rangle \simeq \mathcal{U}_{q t}(\mathfrak{g}),
$$

and that we have the following interpolating property:

$$
\mathcal{U}_{q, 1}(\mathfrak{g}) /\left(\tilde{K}_{i}=1\right) \simeq \mathcal{U}_{q}(\mathfrak{g}) \quad \text { and } \quad \mathcal{U}_{1, t}(\mathfrak{g}) /\left(K_{i}=1\right)=\mathcal{U}_{t}(\mathfrak{g})=\mathcal{U}_{t}\left({ }^{L} \mathfrak{g}\right)
$$

As a special case, we have the elementary interpolating quantum group $\mathcal{U}_{q, t}\left(A_{1}\right)$. The elementary rank one subalgebras of $\mathcal{U}_{q, t}(\mathfrak{g})$ corresponding to simple roots are all isomorphic to $\mathcal{U}_{q, t}\left(A_{1}\right)$ if $\mathfrak{g}$ is simply laced. This is analogous to the properties of standard quantum groups. We will see in the following that for non-simply laced $\mathfrak{g}$ we will have to consider other elementary (rank 1) interpolating quantum groups corresponding to $B_{1}, C_{1}={ }^{L} B_{1}, G_{1}$, and ${ }^{L} G_{1}$. 
3.2 Elementary interpolating quantum groups for $r=2$

For $r=2$ we have $\epsilon=\exp (\pi i / 2)=i$. We will define two elementary interpolating quantum groups $\mathcal{U}_{q, t}\left(C_{1}\right)$ and $\mathcal{U}_{q, t}\left(B_{1}\right)$. The definition of the first one is simple.

Definition $3 \mathcal{U}_{q, t}\left(C_{1}\right)$ is the algebra with generators $X^{ \pm}, K^{ \pm 1}, \tilde{K}^{ \pm 1}$ and relations

$$
\begin{aligned}
K X^{ \pm} & =q^{ \pm 4} X^{ \pm} K, \tilde{K} X^{ \pm}=t^{ \pm 2} X^{ \pm} \tilde{K}, \\
{\left[X^{+}, X^{-}\right] } & =\frac{K \tilde{K}-(K \tilde{K})^{-1}}{q^{2} t-q^{-2} t^{-1}} .
\end{aligned}
$$

Note that

$$
\mathcal{U}_{q, t}\left(C_{1}\right) \supset\left\langle(K \tilde{K})^{ \pm 1}, X^{ \pm}\right\rangle \simeq \mathcal{U}_{q^{2} t}\left(s l_{2}\right)
$$

and that we have the interpolating property

$$
\begin{aligned}
& \mathcal{U}_{q, 1}(\mathfrak{g}) /(\tilde{K}=1) \simeq \mathcal{U}_{q^{2}}\left(s l_{2}\right)=\mathcal{U}_{q}\left(C_{1}\right) \\
& \quad \text { and } \quad \mathcal{U}_{\epsilon, t}(\mathfrak{g}) /(K=1) \simeq \mathcal{U}_{-t}\left(s l_{2}\right)=\mathcal{U}_{-t}\left({ }^{L} C_{1}\right)
\end{aligned}
$$

as $\mathcal{U}_{-t}\left(s l_{2}\right)=\mathcal{U}_{-t}\left(B_{1}\right)$.

Definition $4 \mathcal{U}_{q, t}\left(B_{1}\right)$ is the algebra with generators $X^{ \pm}, K^{ \pm 1}, \tilde{K}^{ \pm 1}, \eta$, central elements $C, \tilde{C}$ and relations

$$
\begin{aligned}
& \mathbb{C}\left[K^{ \pm 1}, \tilde{K}^{ \pm 1}, \eta\right] \text { is commutative, } \\
& K X^{ \pm}=q^{ \pm 2} X^{ \pm} K, \tilde{K} X^{ \pm}=t^{ \pm 1} X^{ \pm} \tilde{K}, \eta X^{ \pm}=X^{ \pm}(\eta \pm 1) \\
& X^{ \pm} X^{\mp}=\frac{q^{C}\left(t^{\tilde{c}} \tilde{K}\right)^{ \pm 1}+q^{-C}\left(t^{\tilde{c}} \tilde{K}^{ \pm 1}\right)^{-P}-q^{\mp 1} t^{ \pm \tilde{c}} \tilde{K} K-q^{ \pm 1} t^{\mp \tilde{c}}(\tilde{K} K)^{-1}}{\left(q-q^{-1}\right)\left(q t-(q t)^{-1}\right)}
\end{aligned}
$$

where $P=(-1)^{\eta}$ and $\tilde{c}=P \tilde{C}-1 / 2$.

Note that we have $t^{\tilde{c}} X^{ \pm}=X^{ \pm} t^{-\tilde{c}-1}, P^{2}=1$ and $P$ commutes with $E^{2}$ and $F^{2}$. We also have the following:

$$
q^{C}\left(t^{\tilde{c}} \tilde{K}^{ \pm 1}\right)^{P}+q^{-C}\left(t^{\tilde{c}} \tilde{K}^{ \pm 1}\right)^{-P}=q^{P C} t^{\tilde{c}} \tilde{K}^{ \pm 1}+q^{-P C} t^{-\tilde{c}} \tilde{K}^{\mp 1}
$$

The elements

$$
\operatorname{Cas}(q)=q^{C}+q^{-C} \text { and } \operatorname{Cas}(t)=t^{\tilde{c}+1 / 2}+t^{-\tilde{c}-1 / 2}
$$

are central. The element $\operatorname{Cas}(q)$ will correspond to the Casimir element for the specialization $t=1$. For the other specialization, $q=\epsilon$, the Casimir element will not be exactly $\operatorname{Cas}(t)$, but $t^{2(1+\tilde{c})}+t^{-2(1+\tilde{c})}$, which is not central in the whole algebra, but commutes with $\left(X^{ \pm}\right)^{2}$. 
Lemma 3 The algebra $\mathcal{U}_{q, t}\left(B_{1}\right)$ is well-defined.

Proof The only point to be checked is the associativity condition

$$
\left(X^{ \pm} X^{\mp}\right) X^{ \pm}=X^{ \pm}\left(X^{\mp} X^{ \pm}\right) .
$$

It is satisfied as we have

$$
\begin{aligned}
& \left(q^{C}\left(t^{\tilde{c}} \tilde{K}^{ \pm 1}\right)^{P}+q^{-C}\left(t^{\tilde{c}} \tilde{K}^{ \pm 1}\right)^{-P}-q^{\mp 1} t^{ \pm \tilde{c}} \tilde{K} K-q^{ \pm 1} t^{\mp \tilde{c}}(\tilde{K} K)^{-1}\right) X^{ \pm} \\
& =X^{ \pm}\left(q^{C}\left(t^{-\tilde{c}} \tilde{K}^{ \pm 1}\right)^{-P}+q^{-C}\left(t^{-\tilde{c}} \tilde{K}^{ \pm 1}\right)^{P}-q^{ \pm 1} t^{\mp \tilde{c}} \tilde{K} K-q^{\mp 1} t^{ \pm \tilde{c}}(\tilde{K} K)^{-1}\right) .
\end{aligned}
$$

Let us look at the specializations of $\mathcal{U}_{q, t}\left(B_{1}\right)$ at $t=1$ and $q=\epsilon=i$. Let

$$
\mathcal{X}^{ \pm}=\mp\left(X^{ \pm}\right)^{2} /\left(t-t^{-1}\right), \quad \mathcal{K}=\tilde{K}^{2} .
$$

Proposition 2 The subalgebra of $\mathcal{U}_{q, 1}\left(B_{1}\right) /(\tilde{K}=1)$ generated by $X^{ \pm}, K^{ \pm 1}$ is isomorphic to $\mathcal{U}_{q}\left(s_{2}\right)=\mathcal{U}_{q}\left(B_{1}\right)$.

The subalgebra of $\mathcal{U}_{\epsilon, t}\left(B_{1}\right) /\left(K^{2}=1, K q^{P C}=\epsilon\right)$ generated by $\mathcal{X}^{ \pm}, \mathcal{K}^{ \pm 1}$ is isomorphic to $\mathcal{U}_{t^{2}}\left(s l_{2}\right)=\mathcal{U}_{-t}\left(C_{1}\right)=\mathcal{U}_{-t}\left({ }^{L} B_{1}\right)$.

Proof First, let us consider the specialization $\mathcal{U}_{q, 1}\left(B_{1}\right)$ at $t=1$. Then the element $\tilde{K}$ becomes central and we can specialize $\tilde{K}=1$. We have the relations $K X^{ \pm}=q^{ \pm 2} X^{ \pm} K$ and

$$
\left(q-q^{-1}\right)^{2} X^{ \pm} X^{\mp}+q^{\mp 1} K+q^{ \pm 1} K^{-1}=\operatorname{Cas}(q) .
$$

The equality implies the standard relation

$$
\left[X^{+}, X^{-}\right]=\frac{K-K^{-1}}{q-q^{-1}} \text {. }
$$

$\operatorname{Cas}(q)$ is central and corresponds to the central Casimir element in $\mathcal{U}_{q}\left(s l_{2}\right)$. So we have an isomorphism.

Now let us consider the specialization of $\mathcal{U}_{q, t}\left(B_{1}\right)$ at $q=\epsilon$. Then $K^{2}$ becomes central. Let us consider the algebra $\mathcal{U}_{\epsilon, t}\left(B_{1}\right) /\left(K^{2}=1\right)$. We have the relations:

$$
\begin{aligned}
K X^{ \pm} & =-X^{ \pm} K, K X^{ \pm}=-X^{ \pm} K, \tilde{K} X^{ \pm}=t^{ \pm 1} X^{ \pm} \tilde{K} \\
X^{ \pm} X^{\mp} & =\frac{\left(q^{P C}+\epsilon K\right)\left(t^{\tilde{c}} \tilde{K}{ }^{ \pm 1}-\epsilon q^{-P C} K t^{-\tilde{c}} \tilde{K} \mp 1\right)}{-2\left(t+t^{-1}\right)} .
\end{aligned}
$$

Since $\left(q^{P C}+\epsilon K\right) X^{ \pm}=X^{ \pm}\left(q^{-P C}-\epsilon K\right)=X^{ \pm}\left(-\epsilon K q^{-P C}\right)\left(q^{P C}+\epsilon K\right)$, we find that $4\left(t+t^{-1}\right)^{2}\left(X^{ \pm}\right)^{2}\left(X^{\mp}\right)^{2}$ is equal to

$$
\begin{aligned}
- & \epsilon q^{-P C}\left(q^{P C} K+\epsilon\right)^{2}\left(t^{-\tilde{c}-2} \tilde{K}^{ \pm 1}+\epsilon q^{P C} K t^{\tilde{c}+2} \tilde{K}^{\mp 1}\right) \\
& \times\left(t^{\tilde{c}} \tilde{K}^{ \pm 1}-\epsilon q^{-P C} K t^{-\tilde{c}} \tilde{K}^{\mp 1}\right) .
\end{aligned}
$$


So it is natural to specialize at $K q^{P C}=\epsilon$. We obtain that

$$
\left(X^{ \pm}\right)^{2}\left(X^{\mp}\right)^{2}=\frac{t^{-2} \tilde{K}^{ \pm 2}+t^{2} \tilde{K}^{\mp 2}-t^{2(1+\tilde{c})}-t^{-2(1+\tilde{c})}}{\left(t+t^{-1}\right)^{2}} .
$$

The above relations can be rewritten as

$$
\left(t^{2}-t^{-2}\right)^{2} \mathcal{X}^{ \pm} \mathcal{X}^{\mp}+t^{\mp 2} \mathcal{K}+t^{ \pm 2} \mathcal{K}^{-1}=t^{2(1+\tilde{c})}+t^{-2(1+\tilde{c})}
$$

The element $t^{2(1+\tilde{c})}+t^{-2(1+\tilde{c})}$ commutes with $\mathcal{X}^{ \pm}, \mathcal{K}^{ \pm 1}$ and corresponds to the Casimir element (see the above discussion). We get the equality

$$
\left[\mathcal{X}^{+}, \mathcal{X}^{-}\right]=\frac{\mathcal{K}-\mathcal{K}^{-1}}{t^{2}-t^{-2}}
$$

\subsection{Interpolating quantum group for $r=2$}

Let $\mathfrak{g}$ be a simple Lie algebra such that $r=2$, that is, $\mathfrak{g}$ is of type $B_{n}, C_{n}$ or $F_{4}$.

Definition $5 \mathcal{U}_{q, t}(\mathfrak{g})$ is the algebra with generators $X_{i}^{ \pm}, K_{i}^{ \pm 1}, \tilde{K}_{i}^{ \pm 1}, \eta_{j}, C_{j}, \tilde{C}_{j}(1 \leq$ $\left.i, j \leq n, r_{j}=1\right)$ and relations

$$
\begin{aligned}
& \mathbb{C}\left[K_{i}^{ \pm}, \tilde{K}_{i}^{ \pm 1}, \eta_{j}, C_{j}, \tilde{C}_{j}\right]_{1 \leq i, j \leq n, r_{j}=1} \text { is commutative, } \\
& \mathcal{U}_{i}=\left\langle X_{i}^{ \pm}, K_{i}^{ \pm 1}, \tilde{K}_{i}^{ \pm 1}\right\rangle \simeq \mathcal{U}_{q, t}\left(C_{1}\right) \text { if } r_{i}=2, \\
& \mathcal{U}_{i}=\left\langle X_{i}^{ \pm}, K_{i}^{ \pm 1}, \tilde{K}_{i}^{ \pm 1}, \eta, C_{i}, \tilde{C}_{i}\right\rangle \simeq \mathcal{U}_{q, t}\left(B_{1}\right) \quad \text { if } r_{i}=1, \\
& K_{i} X_{j}^{ \pm}=q^{ \pm r_{i} C_{i, j}} X_{j}^{ \pm} K_{i}, \quad \tilde{K}_{i} X_{j}^{ \pm}=t^{ \pm r_{i} C_{i, j} / 2} \tilde{K}_{j}, \\
& {\left[X_{i}^{+}, X_{j}^{-}\right]=\left[(-1)^{\eta_{i}}, X_{j}^{ \pm}\right]=0 \text { for } i \neq j .}
\end{aligned}
$$

Let us consider the elements

$$
\begin{aligned}
& \mathcal{X}_{i}^{ \pm}=\mp\left(X_{i}^{ \pm}\right)^{2} /\left(t-t^{-1}\right), \quad \mathcal{K}_{i}=\tilde{K}_{i}^{2} \text { for } r_{i}=1 \\
& \mathcal{X}_{i}^{ \pm}=X_{i}^{ \pm}, \quad \mathcal{K}_{i}=\tilde{K}_{i} \text { for } r_{i}=2 .
\end{aligned}
$$

The specialization at $q=\epsilon=i=\sqrt{-1}$ should not be confused in the following with the index $i \in I$. Proposition 2 implies

Proposition 3 The subalgebra of $\mathcal{U}_{q, 1}(\mathfrak{g}) /\left(\tilde{K}_{i}=1\right)$ generated by the $X_{i}^{ \pm}, K_{i}^{ \pm 1}$ is isomorphic to $\mathcal{U}_{q}(\mathfrak{g})$.

The subalgebra of $\mathcal{U}_{\epsilon, t}(\mathfrak{g}) /\left(K_{i}^{2}=1, K_{i} q^{P_{i} C_{i}}=\epsilon\right)$ generated by the $\mathcal{X}_{i}^{ \pm}, \mathcal{K}_{i}^{ \pm 1}$ is isomorphic to $\mathcal{U}_{-t}\left({ }^{L} \mathfrak{g}\right)$.

In the proposition, by convention, $P_{i} C_{i}=1$ if $r_{i}=2$, that is, the relation $K_{i} q^{P_{i} C_{i}}=$ $\epsilon$ means $K_{i}=1$. 
According to the above proposition, $\mathcal{U}_{q, t}(\mathfrak{g})$ interpolates between $\mathcal{U}_{q}(\mathfrak{g})$ and $\mathcal{U}_{-t}\left({ }^{L} \mathfrak{g}\right)$ the quantum groups without the Serre relations. Is it possible to have an algebra that interpolates between the quantum groups $U_{q}(\mathfrak{g})$ and $U_{-t}\left({ }^{L} \mathfrak{g}\right)$ with the Serre relations? In other words, can one construct a two-parameter deformation of the Serre relations of $U_{q}(\mathfrak{g})$ and $U_{-t}\left({ }^{L} \mathfrak{g}\right)$ ? In this paper we are only interested in finite-dimensional representations. Therefore this question is not important, because finite-dimensional representations of $\mathcal{U}_{q}(\mathfrak{g})$ are the same as those of $\mathcal{U}_{q}(\mathfrak{g})$ (and similarly for $\mathcal{U}_{-t}\left({ }^{L} \mathfrak{g}\right)$ and $\left.U_{-t}\left({ }^{L} \mathfrak{g}\right)\right)$. But for other representations this question becomes important. The examples given below indicate that in the framework of $\mathcal{U}_{q, t}(\mathfrak{g})$ the answer is negative.

In fact, in Example 3 of Sect. 5 we will construct a finite-dimensional representation $V$ of $\mathcal{U}_{q, t}\left(B_{2}\right)$ which interpolates between representations of $\mathcal{U}_{q}\left(B_{2}\right)$ and $\mathcal{U}_{-t}\left(C_{2}\right)$ (and hence of $U_{q}\left(B_{2}\right)$ and $U_{-t}\left(C_{2}\right)$ ), but for different vectors in this representation different $t$-deformations of the Serre relations of $U_{q}\left(B_{2}\right)$ will be satisfied. Imposing either of them (or another $t$-deformation) on the algebra would lead to additional relations that are not satisfied in $V$. Therefore $V$ is not a module over this algebra. Hence it appears impossible to incorporate a two-parameter deformation of the Serre relations into $\mathcal{U}_{q, t}(\mathfrak{g})$ in such a way that Proposition 3 would hold for the quotient, with $\mathcal{U}_{q}(\mathfrak{g})$ and $\mathcal{U}_{-t}\left({ }^{L} \mathfrak{g}\right)$ replaced by $U_{q}(\mathfrak{g})$ and $U_{-t}\left({ }^{L} \mathfrak{g}\right)$.

To illustrate this point further, consider the following example of a candidate for a $t$-deformation of the Serre relations for $\mathfrak{g}=B_{2}$ (note that we do not use it in this paper):

$$
\begin{aligned}
& X_{2}^{+} X_{1}^{+2}-\left(q^{2} t+q^{-2} t^{-1}\right) X_{1}^{+} X_{2}^{+} X_{1}^{+}+X_{1}^{+2} X_{2}^{+}=0 \\
& X_{1}^{+} X_{2}^{+3}-t\left(q^{2}+1+q^{-2}\right) X_{2}^{+} X_{1}^{+} X_{2}^{+2} \\
& \quad+t^{-2}\left(q^{2}+1+q^{-2}\right) X_{2}^{+2} X_{1}^{+} X_{2}^{+}-t^{-1} X_{2}^{+3} X_{1}^{+}=0 .
\end{aligned}
$$

At $t=1$ we recover the Serre relation of $\mathcal{U}_{q}\left(B_{2}\right)$. Let us consider the specializations $S, S^{\prime}$ of these relations at $q=\epsilon$. By computing $S^{\prime} X_{2}^{+}-t X_{2}^{+} S^{\prime}$ we obtain

$$
X_{1}^{+}\left(X_{2}^{+2}\right)^{2}-\left(t^{2}+t^{-2}\right)\left(X_{2}^{+2}\right) X_{1}^{+}\left(X_{2}^{+2}\right)+\left(X_{2}^{+2}\right)^{2} X_{1}^{+}=0
$$

which is one of the Serre relation of $\mathcal{U}_{-t}\left(C_{2}\right)$. By computing

$$
\begin{gathered}
X_{2}^{+} S X_{1}^{+}-\left(t^{2}+1+t^{-2}\right) X_{1}^{+} X_{2}^{+} S+\left(t^{2}+1+t^{-2}\right) S X_{2}^{+} X_{1}^{+} \\
-X_{1}^{+} S X_{2}^{+}-\left(t+t^{-1}\right) X_{2}^{+} X_{1}^{+} S+\left(t+t^{-1}\right) S X_{1}^{+} X_{2}^{+},
\end{gathered}
$$

we obtain

$$
\begin{aligned}
& \left(X_{2}^{+^{2}}\right) X_{1}^{+^{3}}-\left(t^{2}+1+t^{-2}\right) X_{1}^{+}\left(X_{2}^{+^{2}}\right) X_{1}^{+^{2}} \\
& \quad+\left(t^{2}+1+t^{-2}\right) X_{1}^{+^{2}}\left(X_{2}^{+^{2}}\right) X_{1}^{+}-X_{1}^{+3}\left(X_{2}^{+2}\right)=0
\end{aligned}
$$


which is another Serre relation for $\mathcal{U}_{-t}\left(C_{2}\right)$ (both relations should be written in terms of $X_{1}^{+}$and $\left.\mathcal{X}_{2}^{+}=\left(X_{2}^{+}\right)^{2}\right)$.

But if we compute the bracket of the second Serre relation with $X_{1}^{-}$, we obtain

$$
K_{1} X_{2}^{+3} q^{2}\left(1+q^{2}\right)\left(t^{-4}-1\right)=\left(1-t^{2}\right)\left(1-q^{-2}\right)^{2}\left(1+q^{-2}\right) K_{1}^{-1} X_{2}^{+3} .
$$

Then we following identity which does not hold in either $U_{q}\left(B_{2}\right)$ or $\mathcal{U}_{-t}\left(C_{2}\right)$ :

$$
K_{1} X_{2}^{+3} q^{4}\left(1+t^{2}\right)=t^{4}\left(1-q^{-2}\right)^{2} K_{1}^{-1} X_{2}^{+3} .
$$

Hence if we include the relations (7), we obtain an algebra that does not have the desired interpolation property.

\subsection{Interpolating quantum groups for $r=3$}

For $r=3$ we will define two elementary interpolating quantum groups $\mathcal{U}_{q, t}\left(G_{1}\right)$ and $\mathcal{U}_{q, t}\left({ }^{L} G_{1}\right)$. We have $\epsilon=e^{2 \pi i / 6}$.

Definition $6 \mathcal{U}_{q, t}\left({ }^{L} G_{1}\right)$ is the algebra with generators $X^{ \pm}, K^{ \pm 1}, \tilde{K}^{ \pm 1}$ and relations

$$
\begin{aligned}
K X^{ \pm} & =q^{ \pm 6} X^{ \pm} K, \quad \tilde{K} X^{ \pm}=t^{ \pm 2} X^{ \pm} \tilde{K}, \\
{\left[X^{+}, X^{-}\right] } & =\frac{K \tilde{K}-(K \tilde{K})^{-1}}{q^{3} t-q^{-3} t^{-1}} .
\end{aligned}
$$

Note that

$$
\mathcal{U}_{q, t}\left({ }^{L} G_{1}\right) \supset\left\langle(K \tilde{K})^{ \pm 1}, X^{ \pm}\right\rangle \simeq \mathcal{U}_{q^{3} t}\left(s l_{2}\right)
$$

and that we have the following interpolating property:

$$
\begin{aligned}
& \mathcal{U}_{q, 1}(\mathfrak{g}) /(\tilde{K}=1) \simeq \mathcal{U}_{q^{3}}\left(s l_{2}\right)=\mathcal{U}_{q}\left({ }^{L} G_{1}\right) \\
& \quad \text { and } \quad \mathcal{U}_{\epsilon, t}(\mathfrak{g}) /(K=1) \simeq \mathcal{U}_{-t}\left(s l_{2}\right)=\mathcal{U}_{-t}\left(G_{1}\right) .
\end{aligned}
$$

Let us define the elementary interpolating quantum group $\mathcal{U}_{q, t}\left(G_{1}\right)$. First we need the following polynomial map $F(X)=X\left(X-\epsilon^{2}\right)\left(1-\epsilon^{2}\right)^{-1}$ which satisfies $F(1)=1$, $F\left(\epsilon^{2}\right)=0, F\left(\epsilon^{4}\right)=-1$.

Definition 7 We define the algebra $\mathcal{U}_{q, t}\left(G_{1}\right)$ as the algebra with generators $X^{ \pm}, K^{ \pm 1}$, $\tilde{K}^{ \pm 1}, \eta$, central elements $C, \tilde{C}$ and relations

$\mathbb{C}\left[K^{ \pm 1}, \tilde{K}^{ \pm 1}, \eta\right]$ is commutative,

$$
\begin{aligned}
& K X^{ \pm}=q^{ \pm 2} X^{ \pm} K, \tilde{K} X^{ \pm}=t^{ \pm 1} X^{ \pm} \tilde{K}, \quad \eta X^{ \pm}=X^{ \pm}(\eta \pm 1), \\
& X^{ \pm} X^{\mp} \\
& \quad=q^{C}\left(t^{\tilde{c}_{ \pm}} \tilde{K}\right) P_{ \pm}+q^{-C}\left(t^{\tilde{c}_{ \pm}} \tilde{K}\right)^{-P_{ \pm}}-q^{\mp 1} K\left(t^{\tilde{c}_{ \pm}} \tilde{K}\right) P_{ \pm}^{2}-q^{ \pm 1} K^{-1}\left(t^{\tilde{c}_{ \pm}} \tilde{K}\right)^{-P_{ \pm}^{2}},
\end{aligned}
$$

where $P_{ \pm}=F\left(\epsilon^{2(-\eta+1 \mp 1)}\right)$ and $\tilde{c}_{ \pm}=P_{ \pm} \tilde{C} \mp 1 / 2$. 
Lemma 4 The algebra $\mathcal{U}_{q, t}\left(G_{1}\right)$ is well-defined.

Proof The only point to be checked is the associativity condition

$$
\left(X^{ \pm} X^{\mp}\right) X^{ \pm}=X^{ \pm}\left(X^{\mp} X^{ \pm}\right)
$$

which is verified as follows:

$$
\begin{aligned}
& \left(q^{C}\left(t^{\tilde{c}_{ \pm}} \tilde{K}\right)^{P_{ \pm}}+q^{-C}\left(t^{\tilde{c}_{ \pm}} \tilde{K}\right)^{-P_{ \pm}}-q^{\mp 1} K\left(t^{\tilde{c}_{ \pm}} \tilde{K}\right)^{P_{ \pm}^{2}}-q^{ \pm 1} K^{-1}\left(t^{\tilde{c}} \tilde{K}^{-P_{ \pm}^{2}}\right) X^{ \pm}\right. \\
& =X^{ \pm}\left(q^{C}\left(t^{\tilde{c}_{\mp} \mp 1} \tilde{K} t^{ \pm 1}\right)^{P_{\mp}}+q^{-C}\left(t^{\tilde{c}_{\mp \mp 1}} \tilde{K} t^{ \pm 1}\right)^{-P_{\mp}}\right. \\
& \left.-q^{ \pm 1} K\left(t^{\tilde{c}_{\mp} \mp 1} \tilde{K} t^{ \pm 1}\right)^{P_{\mp}^{2}}-q^{\mp 1} K^{-1}\left(t^{\tilde{c}_{\mp} \mp 1} \tilde{K} t^{ \pm 1}\right)^{-P_{\mp}^{2}}\right) \\
& =X^{ \pm}\left(q^{C}\left(t^{\tilde{c}_{\mp}} \tilde{K}\right)^{P_{\mp}}+q^{-C}\left(t^{\tilde{c}_{\mp}} \tilde{K}\right)^{-P_{\mp}}\right. \\
& \left.-q^{ \pm 1} K\left(t^{\tilde{c}_{\mp}} \tilde{K}\right) P_{\mp}^{2}-q^{\mp 1} K^{-1}\left(t^{\tilde{c}_{\mp}} \tilde{K}\right)^{-P_{\mp}^{2}}\right) \text {. }
\end{aligned}
$$

Let us set

$$
\mathcal{X}^{+}=\frac{\left(X^{+}\right)^{3}}{\left(1-\epsilon^{4}\right)^{2}\left(t^{3}-t^{-3}\right)}, \quad \mathcal{X}^{-}=\frac{\left(X^{-}\right)^{3}(-1)^{m+1}}{\left(1+\epsilon^{4}+2 \epsilon^{5}\right)\left(t^{-3}-t^{3}\right)}, \quad \mathcal{K}=\tilde{K}^{2}
$$

Proposition 4 The subalgebra of $\mathcal{U}_{q, 1}\left(G_{1}\right) /(\tilde{K}=1)$ generated by $K^{ \pm 1}, X^{ \pm} /(q-$ $\left.q^{-1}\right)$ is isomorphic to $\mathcal{U}_{q}\left(s_{2}\right)=\mathcal{U}_{q}\left(G_{1}\right)$.

For any $m \in \mathbb{Z} / 2 \mathbb{Z}$, the quotient by $\epsilon^{2 \eta}=1, K=(-1)^{m}, q^{C}=(-1)^{m} \epsilon$ of the subalgebra of $\mathcal{U}_{\epsilon, t}\left(G_{1}\right)$ generated by $\mathcal{X}^{ \pm}, \mathcal{K}$ is isomorphic to $\mathcal{U}_{-t^{3}}\left(s_{2}\right)=\mathcal{U}_{-t}\left({ }^{L} G_{1}\right)$.

Proof The first point is proved as for $\mathcal{U}_{q, 1}\left(B_{1}\right)$. Now let us consider the specialization of $\mathcal{U}_{q, t}\left(G_{1}\right)$ at $q=\epsilon$. Then $K^{3}$ becomes central. Note that we have, $P_{ \pm} X^{\mp}=X^{\mp} P_{0}$, where $P_{0}=F\left(\epsilon^{2-2 \eta}\right)$. We also have $P_{0} X^{ \pm}=X^{ \pm} P_{ \pm}$and

$$
\tilde{c}_{ \pm} X^{\mp}=X^{\mp}\left(P_{0} \tilde{C} \mp 1 / 2\right), \quad\left(P_{0} \tilde{C} \mp 1 / 2\right) X^{\mp}=X^{\mp}\left(\tilde{c}_{\mp} \mp 1\right) .
$$

So we can compute $\left(X^{ \pm}\right)^{3}\left(X^{\mp}\right)^{3}$, and we obtain

$$
\begin{aligned}
& \left(q^{C}\left(t^{\tilde{c}_{ \pm}} \tilde{K}\right)^{P_{ \pm}}+q^{-C}\left(t^{\tilde{c}_{ \pm}} \tilde{K}\right)^{-P_{ \pm}}-q^{\mp 1} K\left(t^{\tilde{c}_{ \pm}} \tilde{K}\right)^{P_{ \pm}^{2}}-q^{ \pm 1} K^{-1}\left(t^{\tilde{c}_{ \pm}} \tilde{K}\right)^{-P_{ \pm}^{2}}\right) \\
& \times\left(q^{C}\left(t^{P_{0} \tilde{C} \mp 3 / 2} \tilde{K}\right)^{P_{0}}+q^{-C}\left(t^{P_{0} \tilde{C} \mp 3 / 2} \tilde{K}\right)^{-P_{0}}\right. \\
& \left.-q^{\mp 3} K\left(t^{P_{0} \tilde{C} \mp 3 / 2} \tilde{K}\right)^{P_{0}^{2}}-q^{ \pm 3} K^{-1}\left(t^{P_{0} \tilde{C} \mp 3 / 2} \tilde{K}\right)^{-P_{0}^{2}}\right) \\
& \times\left(q^{C}\left(t^{\tilde{c}_{\mp} \mp 3} \tilde{K}\right)^{P_{\mp}}+q^{-C}\left(t^{\tilde{c}_{\mp} \mp 3} \tilde{K}\right)^{-P_{\mp}}\right. \\
& \left.-q^{\mp 5} K\left(t^{\tilde{c}_{\mp} \mp 3} \tilde{K}\right)^{P_{\mp}^{2}}-q^{ \pm 5} K^{-1}\left(t^{\tilde{c}_{\mp \mp 3} \tilde{K}}\right)^{-P_{\mp}^{2}}\right) \text {. }
\end{aligned}
$$

Note that $\epsilon^{2 \eta}$ and $K$ commute with $\left(X^{ \pm}\right)^{3}$. So we may take the quotient by the relations $K=(-1)^{m}, \epsilon^{2 \eta}=1$ and $q^{C}=(-1)^{m} \epsilon$ where $m \in \mathbb{Z} / 2 \mathbb{Z}$. In particular, we 
have $P_{0}=0, P_{ \pm}= \pm 1, \tilde{c}_{ \pm}= \pm \tilde{C} \mp 1 / 2$. For $\left(X^{ \pm}\right)^{3}\left(X^{\mp}\right)^{3}$ we obtain

$$
\begin{aligned}
(1+ & \left.\epsilon^{4}+2 \epsilon^{5}\right)\left(t^{ \pm \tilde{c}_{ \pm}} \tilde{K}^{ \pm 1}+\epsilon^{4} t^{\mp \tilde{c}_{ \pm}} \tilde{K}^{\mp 1}-\epsilon^{2 \pm 2} t^{\mp \tilde{c}_{ \pm}} \tilde{K}^{ \pm 1}-\epsilon^{2 \mp 2} t^{ \pm \tilde{c}_{ \pm}} \tilde{K}^{\mp 1}\right) \\
& \times(-1)^{m+1}\left(t^{\mp \tilde{c}_{\mp}+3} \tilde{K}^{\mp 1}+\epsilon^{4} t^{ \pm \tilde{c}_{\mp}-3} \tilde{K}^{ \pm 1}-\epsilon^{2 \mp 2} t^{\mp} \tilde{c}_{\mp 3} \tilde{K}-\epsilon^{2 \pm 2} t^{\mp 3-\tilde{c}_{\mp}} \tilde{K}^{-1}\right) \\
= & (-1)^{m+1}\left(1+\epsilon^{4}+2 \epsilon^{5}\right)\left(1-\epsilon^{4}\right)^{2}\left(t^{\mp \tilde{c}_{\mp}} \tilde{K}^{ \pm 1}-t^{\mp \tilde{c}_{ \pm}} \tilde{K}^{\mp 1}\right) \\
& \times\left(t^{\mp \tilde{c}_{\mp}+3} \tilde{K}^{\mp 1}-t^{ \pm \tilde{c}_{\mp}-3} \tilde{K}^{ \pm 1}\right) \\
= & (-1)^{m+1}\left(1+\epsilon^{4}+2 \epsilon^{5}\right)\left(1-\epsilon^{4}\right)^{2}\left(-t^{-3} \tilde{K}^{ \pm 2}-t^{3} \tilde{K}^{\mp 2}+t^{2 \tilde{C}+2}+t^{-2 \tilde{C}-2}\right) .
\end{aligned}
$$

We have

$$
-\mathcal{X}^{ \pm} \mathcal{X}^{\mp}\left(t^{3}-t^{-3}\right)^{2}+t^{\mp 3} \tilde{K}^{2}+t^{ \pm 3} \tilde{K}^{-2}=t^{2 \tilde{C}+2}+t^{-2 \tilde{C}-2}
$$

Now we are in position to define the interpolating quantum group $\mathcal{U}_{q, t}\left(G_{2}\right)$.

Definition $8 \mathcal{U}_{q, t}\left(G_{2}\right)$ is the algebra with generators $X_{i}^{ \pm}, K_{i}^{ \pm 1}, \tilde{K}_{i}^{ \pm 1}, \eta, C, \tilde{C}(i=$ $1,2)$ and relations $(i \neq j)$

$$
\begin{aligned}
& \mathbb{C}\left[K_{i}^{ \pm}, \tilde{K}_{i}^{ \pm 1}, \eta, C, \tilde{C}\right]_{i=1,2} \text { is commutative, } \\
& \mathcal{U}_{1}=\left\langle X_{1}^{ \pm}, K_{1}^{ \pm 1}, \tilde{K}_{1}^{ \pm 1}\right\rangle \simeq \mathcal{U}_{q, t}\left({ }^{L} G_{1}\right), \\
& \mathcal{U}_{2}=\left\langle X_{2}^{ \pm}, K_{2}^{ \pm 1}, \tilde{K}_{2}^{ \pm} 1, \eta, C, \tilde{C}, \tilde{C}^{\prime}\right\rangle \simeq \mathcal{U}_{q, t}\left(G_{1}\right), \\
& K_{i} X_{j}^{ \pm}=q^{ \pm r_{i} C_{i, j}} X_{j}^{ \pm} K_{i}, \quad \tilde{K}_{i} X_{j}^{ \pm}=t^{ \pm r_{i} C_{i, j} / 3} \tilde{K}_{j}, \\
& {\left[X_{1}^{ \pm}, X_{2}^{\mp}\right]=0 .}
\end{aligned}
$$

We define $\mathcal{X}_{2}^{ \pm}, \mathcal{K}_{2}$ as for $\mathcal{U}_{q, t}\left(G_{1}\right)$. Let $m \in \mathbb{Z} / 2 \mathbb{Z}$. From the above results we have the following:

Proposition 5 The subalgebra of $\mathcal{U}_{q, 1}\left(G_{2}\right) /\left(\tilde{K}_{i}=1\right)$ generated by $X_{1}^{ \pm}, X_{2}^{ \pm} /(q-$ $\left.q^{-1}\right), K_{i}^{ \pm 1}$ is isomorphic to $\mathcal{U}_{q}\left(G_{2}\right)$.

The quotient by $\epsilon^{2 \eta}=1, K_{1}=-1, K_{2}=(-1)^{m}, q^{C}=(-1)^{m} \epsilon$ of the subalgebra of $\mathcal{U}_{\epsilon, t}\left(G_{2}\right)$ generated by $X_{1}^{ \pm}, \mathcal{X}_{2}^{ \pm}, \tilde{K}_{1}^{ \pm 1}, \mathcal{K}_{2}^{ \pm 1}$ is isomorphic to $\mathcal{U}_{-t}\left({ }^{L} G_{2}\right)$.

We have thus defined an interpolating quantum group $\mathcal{U}_{q, t}(\mathfrak{g})$ for any simple Lie algebra $\mathfrak{g}$. The same definition gives us such an algebra for any symmetrizable Kac-Moody algebra $\mathfrak{g}$ such that $r \leq 3$. We just use the relations of the elementary interpolating quantum groups in the same way as above. We conjecture that this definition may also be generalized to all symmetrizable Kac-Moody algebra $\mathfrak{g}$.

\section{Representations of elementary interpolating quantum groups}

Representation theory of the elementary interpolating quantum groups $\mathcal{U}_{q, t}\left(A_{1}\right)$, $\mathcal{U}_{q, t}\left(C_{1}\right), \mathcal{U}_{q, t}\left({ }^{L} G_{1}\right)$ is easily derived from the representation theory of the corresponding standard quantum groups. So we need to consider only $\mathcal{U}_{q, t}\left(B_{1}\right)$ and $\mathcal{U}_{q, t}\left(G_{1}\right)$. 
For these algebras we will observe the simplest examples of representations interpolating between finite-dimensional representations of Langlands dual quantum groups of rank one. Even though naively we have ${ }^{L} B_{1}=B_{1}$ and ${ }^{L} G_{1}=G_{1}$, the resulting duality of representations is non-trivial.

\subsection{Simple finite-dimensional representations of $\mathcal{U}_{q, t}\left(B_{1}\right)$}

As in the representation theory of quantum groups, let us start with Verma modules. We want to construct a Verma module $M(\lambda)$ with respective highest eigenvalues of $(K, \tilde{K}, \eta, C, \tilde{C})$ given by $\lambda=(\lambda, \tilde{\lambda}, E, A, \tilde{A}) \in\left(\mathbb{C}^{*}\right)^{2} \times\{ \pm 1\} \times \mathbb{C}^{2}$. We set:

$$
M(\lambda)=\bigoplus_{n \geq 0} \mathbb{C}\left(X^{-}\right)^{n} v_{\lambda}
$$

We have the obvious action

$$
\begin{aligned}
X^{-} .\left(\left(X^{-}\right)^{n} . v_{\lambda}\right) & =\left(X^{-}\right)^{n+1} . v_{\lambda}, \quad C=A \mathrm{Id}, \quad \tilde{C}=\tilde{A} \mathrm{Id}, \\
K\left(\left(X^{-}\right)^{n} v_{\lambda}\right) & =\lambda q^{-2 n}\left(\left(X^{-}\right)^{n} v_{\lambda}\right), \quad \tilde{K}\left(\left(X^{-}\right)^{n} v_{\lambda}\right)=\tilde{\lambda} t^{-n}\left(\left(X^{-}\right)^{n} v_{\lambda}\right), \\
\eta\left(\left(X^{-}\right)^{n} v_{\lambda}\right) & =(-1)^{n} X^{+}\left(X^{-}\right)^{n} v_{\lambda} .
\end{aligned}
$$

The point is to have a well-defined action of $X^{+}$such that $X^{+} v_{\lambda}=0$. From the relation involving $X^{+} X^{-}$, the action is uniquely defined. The relation involving $X^{-} X^{+}$ evaluated on $v_{\lambda}$ imposes the following condition on $\lambda$ :

Lemma 5 The Verma module $M(\lambda)$ is non-trivial if and only if

$$
q^{E A} t^{E \tilde{A}-\frac{1}{2}} \tilde{\lambda}^{-1}+q^{-E A} t^{-E \tilde{A}+\frac{1}{2}} \tilde{\lambda}-q t^{-E \tilde{A}+\frac{1}{2}} \tilde{\lambda} \lambda-q^{-1} t^{E \tilde{A}-\frac{1}{2}}(\tilde{\lambda} \lambda)^{-1}=0 .
$$

Now we want to have a Verma module with a finite-dimensional quotient. For the specializations defined above, we consider $p \geq 0, n=2 p, \lambda=q^{n}, \tilde{\lambda}=t^{p}$. So we obtain the relation

$$
q^{E A} t^{E \tilde{A}-\frac{1}{2}-p}+q^{-E A} t^{-E \tilde{A}+\frac{1}{2}+p}-q^{1+n} t^{-E \tilde{A}+\frac{1}{2}+p}-q^{-1-n} t^{E \tilde{A}-\frac{1}{2}-p}=0 .
$$

Thus, we have $E A=-(n+1)$ or $(E A=(n+1)$ and $E \tilde{A}=p+1 / 2)$. But to have the second specialization of Proposition 2, we must have $K q^{P C}=\epsilon$ at $q=\epsilon$, so $\epsilon^{n+E A}=\epsilon$. So we are in the second case $E A=n+1$. Consider an $(n+1)$-dimensional vector space

$$
V_{n}=\mathbb{C} v_{0} \oplus \mathbb{C} v_{1} \oplus \cdots \oplus \mathbb{C} v_{n}
$$

We denote $n=2 p \in 2 \mathbb{Z}, v_{-1}=v_{n+1}=0$ and use the usual quantum number notation $[m]_{x}=\left(x^{m}-x^{-m}\right) /\left(x-x^{-1}\right)$ for $m \in \mathbb{Z}$. 
Let us consider operators on $V_{n}$ defined by $C=n+1, \tilde{C}=p+1 / 2$, and the following formulas:

$$
\begin{aligned}
X^{+} v_{2 j} & =[n-2 j+1]_{q} v_{2 j-1}, \quad X^{+} v_{2 j+1}=[n-2 j]_{q t} v_{2 j}, \\
X^{-} v_{2 j} & =[2 j+1]_{q} v_{2 j+1}, \quad X^{-} v_{2 j+1}=[2 j+2]_{q t} v_{2 j+2}, \\
K . v_{j} & =q^{n-2 j} v_{j}, \quad \tilde{K} v_{j}=t^{p-j} v_{j}, \quad \eta . v_{j}=-j v_{j} .
\end{aligned}
$$

The idea of this deformation is just to replace the quantum box $[m]_{q}$ where $m$ is even by $[m]_{q t}$.

Lemma 6 The above formulas define an action of $\mathcal{U}_{q, t}\left(B_{1}\right)$ on $V_{n}$.

Proof All relations are clear without computation, except for relations (6). Let us check these relations.

$$
\begin{aligned}
& \left(q-q^{-1}\right)\left(q t-(q t)^{-1}\right) X^{+} X^{-} \cdot v_{2 j}=\left(q^{2 j+1}-q^{-2 j-1}\right)\left((q t)^{n-2 j}-(q t)^{2 j-n}\right) v_{2 j} \\
& =\left(q^{n+1} t^{n-2 j}+q^{-n-1} t^{2 j-n}-q^{n-4 j-1} t^{n-2 j}-q^{4 j+1-n} t^{2 j-n}\right) v_{2 j}, \\
& \left(q-q^{-1}\right)\left(q t-(q t)^{-1}\right) X^{-} X^{+} \cdot v_{2 j} \\
& =\left(q^{n-2 j+1}-q^{-n+2 j-1}\right)\left((q t)^{2 j}-(q t)^{-2 j}\right) v_{2 j} \\
& =\left(q^{n+1} t^{2 j}+q^{-n-1} t^{-2 j}-q^{n+1-4 j} t^{-2 j}-q^{4 j-n-1} t^{2 j}\right) v_{2 j}, \\
& \left(q-q^{-1}\right)\left(q t-(q t)^{-1}\right) X^{+} X^{-} \cdot v_{2 j+1} \\
& =\left((q t)^{2 j+2}-(q t)^{-2 j-2}\right)\left(q^{n-2 j-1}-q^{2 j-n+1}\right) v_{2 j+1} \\
& =\left(q^{-n-1} t^{-2 j-2}+q^{n+1} t^{2 j+2}-q^{n-4 j-3} t^{-2 j-2}-q^{4 j+3-n} t^{2+2 j}\right) v_{2 j+1}, \\
& \left(q-q^{-1}\right)\left(q t-(q t)^{-1}\right) X^{-} X^{+} \cdot v_{2 j+1} \\
& =\left((q t)^{n-2 j}-(q t)^{-n+2 j}\right)\left(q^{2 j+1}-q^{-2 j-1}\right) v_{2 j+1} \\
& =\left(q^{-n-1} t^{2 j-n}+q^{n+1} t^{n-2 j}-q^{n-1-4 j} t^{n-2 j}-q^{4 j-n+1} t^{2 j-n}\right) v_{2 j+1} .
\end{aligned}
$$

The formulas are also satisfied at the limits as for $X^{-} X^{+} v_{0}$ and for $X^{+} X^{-} v_{2 p}$ we get $q^{n+1}+q^{-n-1}-q^{n+1}-q^{-n-1}=0$.

Note that $V_{n}^{\prime}=\mathbb{C} v_{0} \oplus \mathbb{C} v_{2} \oplus \cdots \oplus \mathbb{C} v_{n}$ is stable for the action of $E^{2}$ and $F^{2}$. We interpret this as a Langlands duality of representations corresponding to $B_{1}$ and $C_{1}$ as follows: the first specialization of $V_{n}$ is the $(n+1)$-dimensional simple representation of $\mathcal{U}_{q}\left(s l_{2}\right)=\mathcal{U}_{q}\left(B_{1}\right)$, and by using the second specialization we obtain $V_{n}^{\prime}$, the $(p+1)$-dimensional representation of $\mathcal{U}_{t^{2}}\left(s l_{2}\right)=\mathcal{U}_{-t}\left(C_{1}\right)=\mathcal{U}_{-t}\left({ }^{L} B_{1}\right)$ (at $q=\epsilon$, we have $K^{2}=1$ and $K q^{P C}=\epsilon$ on $V_{n}$ as in Proposition 2).

Remark 5 If $n \notin 2 \mathbb{Z}$, we can also define a representation of $\mathcal{U}_{q, t}\left(B_{1}\right)$ with the same formulas. Indeed the formulas are also satisfied at the limit: it is the same for $X^{-} X^{+} v_{0}$ and for $X^{+} X^{-} v_{2 j+1}$ where $n=2 j+1$ we get $q^{-n-1} t^{-n-1}+q^{n+1} t^{n+1}-q^{-n-1} t^{-n-1}-$ $q^{n+1} t^{n+1}=0$. But then we cannot use the second specialization on $V_{n}^{\prime}$ at $q=\epsilon$ as we have $K q^{P C} v_{0}=-v_{0}$ different than in Proposition 2. 


\subsection{Representations of $\mathcal{U}_{q, t}\left(G_{1}\right)$}

Let $V_{n}$ be as in Sect. 4.1 where $n \in 3 \mathbb{Z}$. Let us consider operators defined by $C=n+1$, $\tilde{C}=(n+1) / 2$ and the following formulas:

$$
\begin{aligned}
X^{+} v_{3 j} & =\left(q-q^{-1}\right)[n-3 j+1]_{q} v_{3 j-1}, \quad X^{-} v_{3 j}=\left(q-q^{-1}\right)[3 j+1]_{q} v_{3 j+1}, \\
X^{+} v_{3 j+1} & =\left(q t-(q t)^{-1}\right)[n-3 j]_{q t} v_{3 j}, \quad X^{-} v_{3 j+1}=\left(q-q^{-1}\right)[3 j+2]_{q} v_{3 j+2}, \\
X^{+} v_{3 j+2} & =\left(q-q^{-1}\right)[n-3 j-1]_{q} v_{3 j+1}, \\
X^{-} v_{3 j+2} & =\left(q t-(q t)^{-1}\right)[3 j+3]_{q t} v_{3 j+2}, \\
K . v_{j} & =q^{n-2 j} v_{j}, \quad \tilde{K} v_{j}=t^{n / 2-j} v_{j}, \quad \eta \cdot v_{j}=-j v_{j} .
\end{aligned}
$$

The idea of this deformation is just to replace the quantum box $[m]_{q}$ where $m \equiv 0[3]$ by $[m]_{q t}$. This is analog to the deformation considered for $B_{1}$.

Note that we have in particular

$$
\begin{aligned}
& P_{+} . v_{3 j}=v_{3 j}, \quad P_{+} . v_{3 j+1}=0, \quad P_{+} . v_{3 j+2}=-v_{3 j+2}, \\
& P_{-} . v_{3 j}=-v_{3 j}, \quad P_{-} . v_{3 j+1}=v_{3 j+1}, \quad P_{-} . v_{3 j+2}=0 .
\end{aligned}
$$

Lemma 7 The above formulas define an action of $\mathcal{U}_{q, t}\left(G_{1}\right)$ on $V_{n}$.

Proof All relations are clear without computation, except for relations (8). Let us check these relations.

$$
\begin{aligned}
X^{+} X^{-} . v_{3 j} & =\left(q^{3 j+1}-q^{-3 j-1}\right)\left((q t)^{n-3 j}-(q t)^{3 j-n}\right) v_{3 j} \\
& =\left(q^{n+1} t^{n-3 j}+q^{-n-1} t^{3 j-n}-q^{n-6 j-1} t^{n-3 j}-q^{6 j+1-n} t^{3 j-n}\right) v_{3 j}, \\
X^{+} X^{-} . v_{3 j+1} & =\left(q^{3 j+2}-q^{-3 j-2}\right)\left(q^{n-3 j-1}-q^{3 j-n+1}\right) v_{3 j+1} \\
& =\left(q^{n+1}+q^{-n-1}-q^{n-6 j-3}-q^{6 j+3-n}\right) v_{3 j+1}, \\
X^{+} X^{-} . v_{3 j+2} & =\left((q t)^{3 j+3}-(q t)^{-3 j-3}\right)\left(q^{n-3 j-2}-q^{3 j-n+2}\right) v_{3 j+2} \\
& =\left(q^{n+1} t^{3 j+3}+q^{-n-1} t^{-3 j-3}-q^{n-6 j-5} t^{-3 j-3}-q^{6 j+5-n} t^{3 j+3}\right) v_{3 j+2}, \\
X^{-} X^{+} . v_{3 j} & =\left(q^{n-3 j+1}-q^{3 j-1-n}\right)\left((q t)^{3 j}-(q t)^{-3 j}\right) v_{3 j} \\
& =\left(q^{n+1} t^{3 j}+q^{-n-1} t^{-3 j}-q^{n-6 j+1} t^{-3 j}-q^{6 j-1-n} t^{3 j}\right) v_{3 j}, \\
X^{-} X^{+} . v_{3 j+1} & =\left((q t)^{n-3 j}-(q t)^{3 j-n}\right)\left(q^{3 j+1}-q^{-3 j-1}\right) v_{3 j+1} \\
& =\left(q^{n+1} t^{n-3 j}+q^{-n-1} t^{3 j-n}-q^{n-6 j-1} t^{n-3 j}-q^{6 j+1-n} t^{3 j-n}\right) v_{3 j+1}, \\
X^{-} X^{+} . v_{3 j+2} & =\left(q^{n-3 j-1}-q^{3 j+1-n}\right)\left(q^{3 j+2}-q^{-3 j-2}\right) v_{3 j+2} \\
& =\left(q^{n+1}+q^{-n-1}-q^{n-6 j-3}-q^{6 j+3-n}\right) v_{3 j+2} .
\end{aligned}
$$

The formulas are also satisfied at the limits as for $X^{-} X^{+} v_{0}$ and also for $X^{+} X^{-} v_{3(n / 3)}$ we get $q^{n+1}+q^{-n-1}-q^{n+1}-q^{-n-1}=0$. 
Note that $V_{n}^{\prime}=\mathbb{C} v_{0} \oplus \mathbb{C} v_{3} \oplus \cdots \oplus \mathbb{C} v_{n}$ is stable for the action of $E^{3}$ and $F^{3}$. We interpret this as a Langlands duality of representations of $G_{1}$ : by using the first specialization, $V_{n}$ becomes the $(n+1)$-dimensional simple representation of $\mathcal{U}_{q}\left(s l_{2}\right)=\mathcal{U}_{q}\left(G_{1}\right)$, and by using the second specialization we obtain $V_{n}^{\prime}$, the $n / 3+$ 1-dimensional representation of $\mathcal{U}_{t^{3}}\left(s l_{2}\right)=\mathcal{U}_{t}\left({ }^{L} G_{1}\right)$ (at $q=\epsilon$, we have $\epsilon^{\eta}=1$, $K=(-1)^{n / 3}$ and $q^{C}=(-1)^{n / 3} \epsilon$ on $V_{n}^{\prime}$ as in Proposition 4$)$.

Remark 6 If $n \notin 3 \mathbb{Z}$, we can also define a representation of $\mathcal{U}_{q, t}\left(B_{1}\right)$ by the same formulas. Indeed, the formulas are also satisfied at the limit: it is the same for $X^{-} X^{+} v_{0}$, for $X^{+} X^{-} v_{3 j+1}$ where $n=3 j+1$ we get $q^{n+1}+q^{-n-1}-q^{-n-1}-q^{n+1}=0$, and for $X^{+} X^{-} v_{3 j+2}$ where $n=3 j+2$ we get $q^{n+1} t^{n+1}+q^{-n-1} t^{-n-1}-q^{-n-1} t^{-n-1}-$ $q^{n+1} t^{n+1}=0$. But then we cannot use the second specialization on $V_{n}^{\prime}$ as at $q=\epsilon$ we have $K q^{C} v_{0}=\epsilon^{2 n+1} v_{0}$ different than in Proposition 4.

\subsection{Another interpretation of the duality}

In this section we discuss an interpretation of the Langlands dual of Sect. 4.1 in terms of the structure of the algebra $\mathcal{U}_{q, t}\left(B_{1}\right)$.

The duality of the simple finite dimensional representations of $\mathcal{U}_{q, t}\left(B_{1}\right)$ in Sect. 4.1 in terms of characters is just the elementary duality between the following polynomials:

$$
y^{2 n}+y^{2 n-2}+\cdots+y^{2-2 n}+y^{-2 n} \leftrightarrow\left(y^{2}\right)^{n}+\left(y^{2}\right)^{n-2}+\cdots+\left(y^{2}\right)^{2-n}+\left(y^{2}\right)^{-n} .
$$

We have seen that it corresponds to an interpolating representation. At the level of characters, we can define a similar interpolation. Indeed let us consider a map $\alpha(q, t)$ such that $\alpha(q, 1)=1$ and $\alpha(\epsilon, t)=0$. Such a map is naturally introduced in $[10,11]$ (we will also see an elementary way to introduce it bellow):

$$
\alpha(q, t)=\left(q+q^{-1}\right)\left(q t-q^{-1} t^{-1}\right)\left(q^{2} t-q^{-2} t^{-1}\right)^{-1}
$$

In the following it will just be denoted by $\alpha$. Then the character

$$
y^{2 n}+\alpha y^{2 n-2}+y^{2 n-4}+\alpha y^{2 n-6}+\cdots+y^{4-2 n}+\alpha y^{2-2 n}+y^{-2 n}
$$

interpolates between the two formulas.

The character of a fundamental representation is $y+y^{-1}$. This corresponds to the decomposition of the Casimir central elements

$$
\operatorname{Cas}(q)=q^{C}+q^{-C}, \quad \operatorname{Cas}(t)=t^{\tilde{c}+1 / 2}+t^{-\tilde{c}-1 / 2} .
$$


The Casimir element of the second specialization is $\left(t^{2(\tilde{c}+1)}+t^{-2(\tilde{c}+1)}\right)$, so we have the following picture:

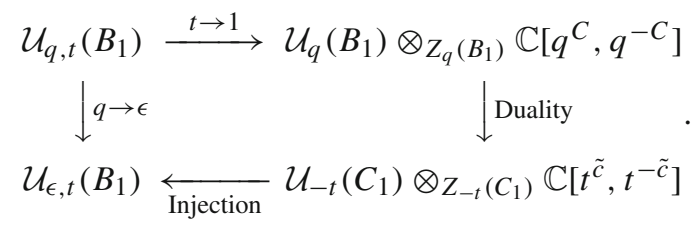

Note that the tensor product $\mathcal{U}_{q}\left(B_{1}\right) \otimes_{Z_{q}\left(B_{1}\right)} \mathbb{C}\left[q^{C}, q^{-C}\right]$ is a quantum analogue (for $\mathfrak{g}=B_{1}$ ) of the algebras

$$
U(\mathfrak{g}) \otimes_{Z(U(\mathfrak{g}))} U(\mathfrak{h})
$$

where $\mathfrak{h} \subset \mathfrak{g}$ is the Cartan subalgebra, considered by Gelfand and Kirillov [13].

The decomposition of the Casimir element and the character formulas are closely related. This can be put a step forward by having a similar interpretation of the interpolating character in the spirit of the constructions of $[10,11]$ in the affine case. Indeed, we have the central element $Y+Y^{-1}$ in $\mathcal{U}_{q, t}\left(B_{1}\right)$ where $Y=q^{P C} t^{\tilde{c}+1 / 2}$, which interpolates between $\operatorname{Cas}(q)$ and $\operatorname{Cas}(t)$. (Note that we have $Y^{ \pm 1} X^{+}=X^{+} Y^{\mp 1}$.)

We define the completed algebra $\tilde{\mathcal{U}}_{q, t}\left(B_{1}\right)$ as the algebra containing $\mathcal{U}_{q, t}\left(B_{1}\right)$ with additional elements $W^{ \pm 1}$ such that $W^{ \pm 1} X^{+}=X^{+} W^{\mp 1}$ and $Y W=\alpha W Y$. Note that because of the relation of the algebra, we cannot require it to commute with $X^{-}$. Note also that we have $Y^{-1} W^{-1}=\alpha W^{-1} Y^{-1}$ which is compatible with the commutation relations with $X^{+}$.

Let us explain how such a commutation relation $Y W=\alpha W Y$ can be obtained naturally in the spirit of $[10,11]$. We recall that the variables for the $q$-characters (affine version of characters) are materialized as formal power series in generators of the Heisenberg subalgebra of the level 0 quantum affine algebra. The $q, t$-analogues of these variables (which are the building blocks for the generating series of the deformed $\mathcal{W}$-algebra $\left.\mathcal{W}_{q, t}(\mathfrak{g})\right)$ are, in turn, related to a non-commutative Heisenberg algebra. In the $B_{1}$-case this Heisenberg algebra has generators $h[n](n \in \mathbb{Z})$ such that $h[0]$ is central and for $n, m \neq 0$ we have

$$
[h[n], h[m]]=\delta_{n,-m} \frac{\left(q^{n}-q^{-n}\right)\left(t^{n}-t^{-n}\right)}{n} .
$$

As the 0th mode $h[0]$ is central, it is not clear how to obtain the commutation relations as considered above. But the finite type can also be seen as a limit of the affine type case in the following sense. Let

$$
h_{ \pm}(z)=\exp \left(\sum_{ \pm m \geq 0} h[m] z^{-m}\right)
$$


Then $h_{+}\left(z q^{3} t\right) h_{-}(w)$ is equal to

$$
\begin{aligned}
& h_{-}(w) h_{+}\left(z q^{3} t\right) \exp \left(\sum_{m>0} \frac{\left(q^{-2 m}-q^{-4 m}\right)\left(1-t^{-2 m}\right)}{m}\left(w z^{-1}\right)^{m}\right) \\
& =h_{-}(w) h_{+}\left(z q^{3} t\right) \frac{\left(1-q^{-4} w z^{-1}\right)\left(1-t^{-2} q^{-2} w z^{-1}\right)}{\left(1-q^{-4} t^{-2} w z^{-1}\right)\left(1-q^{-2} w z^{-1}\right)}
\end{aligned}
$$

A priori, we cannot directly specialize at $z=w=1$. But if we forget the intermediate formulas, everything makes sense for this specialization, and for $Y=h_{+}\left(q^{3} t\right)$, $W=h_{-}(1)$ we obtain

$$
Y W=\frac{\left(1-q^{-4}\right)\left(1-t^{-2} q^{-2}\right)}{\left(1-q^{-4} t^{-2}\right)\left(1-q^{-2}\right)} W Y=\alpha W Y .
$$

To give a precise meaning to this specialization, we consider an additional formal parameter $u$ and replace $w, z$, respectively, by $w u, z u^{-1}$. We get formal power series in $u^{-1}$. So we can set $z=w=1$ and for $Y(u)=h_{+}\left(u^{-1} q^{3} t\right)$ and $W(u)=h_{-}(u)$ we obtain

$$
Y(u) W(u)=\frac{\left(1-q^{-4} u^{2}\right)\left(1-t^{-2} u^{2}\right)}{\left(1-q^{-2} u^{2}\right)\left(1-q^{-4} t^{-2} u^{2}\right)} W(u) Y(u)
$$

Now we can specialize from the affine type to the finite type by considering $Y=Y(1)$, $W=W(1)$, and we get $Y W=\alpha W Y$ as explained above.

We have a notion of normal ordering : $M$ : for monomials $M$ in $Y^{ \pm 1}, W^{ \pm 1}$, where we put the $Y^{ \pm 1}$ on the left and the $W^{ \pm 1}$ on the right. Then we have

$$
\begin{aligned}
\left(: Y W+Y^{-1} W^{-1}:\right)^{2} & =\alpha^{-1}:(Y W)^{2}:+2 \alpha+\alpha^{-1}:\left(Y^{-1} W^{-1}\right)^{2}: \\
& =\alpha^{-1}\left(:(Y W)^{2}:+\alpha^{2}+:\left(Y^{-1} W^{-1}\right)^{2}:\right)+\alpha .
\end{aligned}
$$

In particular, the formula corresponding to the three-dimensional simple representation appears naturally as

$$
:(Y W)^{2}:+\alpha^{2}+:\left(Y^{-1} W^{-1}\right)^{2}:
$$

Note that this formula commutes with $X^{+}$, as does : $Y W+Y^{-1} W^{-1}$ :, which has the same property. This can be interpreted as an analog of the invariance of the usual characters for the Weyl group action or of the symmetry property of $q$-characters for the screening operators (see $[11,12]$ ).

It would be desirable to have a similar interpretation of the duality of characters for general quantum groups. 


\section{More general interpolating representations}

By an interpolating representation we understand a representation of the interpolating quantum group $\mathcal{U}_{q, t}(\mathfrak{g})$ which gives by specialization representations of the Langlands dual quantum groups. We have seen in the Sect. 4 that interpolating representations exist for elementary interpolating quantum groups. In this section we give additional examples for non-elementary interpolating quantum groups. We believe that any irreducible representation $L(\lambda)$ of $U_{q}(\mathfrak{g})$ (equivalently, of $\mathcal{U}_{q}(\mathfrak{g})$ ) with $\lambda \in P^{\prime}$ may be $t$-deformed, in an essentially unique way, to a representation of $\mathcal{U}_{q, t}(\mathfrak{g})$ in such a way that its specialization at $q=\epsilon$ gives a representation of $U_{-t}\left({ }^{L} \mathfrak{g}\right)$ whose character is $\Pi(\chi(\lambda))$.

We start with a simple finite-dimensional representation $V$ of $\mathcal{U}_{q}\left(B_{2}\right)$ with highest weight which has an even multiplicity for the node 2 . We want to "deform" the $\mathcal{U}_{q}\left(B_{2}\right)$-module structure on $V$. All weights of $V$ have even multiplicities for the node 2. For $v \in V$ of weight $m_{1} \omega_{1}+2 m_{2} \omega_{2}$, we set

$$
K_{2} v=q^{2 m_{2}} v, \quad \tilde{K}_{2} v=t^{m_{2}} v, \quad K_{1} v=q^{2 m_{1}} v, \quad \tilde{K}_{1} v=t^{m_{1}} v
$$

The deformation will be necessarily semi-simple for $\mathcal{U}_{1} \simeq \mathcal{U}_{q^{2} t}\left(A_{1}\right)$, but moreover we require that it is semi-simple for the action of $\mathcal{U}_{2}=\mathcal{U}_{q, t}\left(B_{1}\right)$ with simple submodules isomorphic to the representations constructed in Sect. 4. The actions of $C$ and $\tilde{C}$ are uniquely determined from the action of $X_{2}^{+}$and $X_{2}^{-}$as it suffices to know the decomposition in simple modules for $\mathcal{U}_{2}$. So the non-trivial point is to deform the action of the $X_{i}^{+}, X_{i}^{-}$.

We will consider 3 examples of interpolating representations of $\mathcal{U}_{q, t}\left(B_{2}\right)$. At the level of crystals, they correspond to the examples studied in Sect. 2.1. The first one is the most simple example where the duality occurs. In the second example we have a multiplicity in the character and we can see that the relations between $C$ and the $X_{1}^{ \pm}$cannot be written a simple way. In the third example we observe that different $t$-deformations of the Serre relations arise in the interpolating representations.

Example 1 Let $V=L\left(\omega_{1}\right)$ be the fundamental representation of $\mathcal{U}_{q}\left(B_{2}\right)$ of dimension 5 which corresponds by duality to the representation of $\mathcal{U}_{-t}\left(C_{2}\right)$ whose highest component is the fundamental representation of dimension 4. Its character is $y_{1}+$ $y_{2}^{2} y_{1}^{-1}+1+y_{1} y_{2}^{-2}+y_{1}^{-1}$, and all weight spaces are of dimension 1 .

We consider a basis $\left(v_{l}\right)_{1 \leq l \leq 5}$ of $V$ such that $v_{1}$ is a highest weight vector,

$$
v_{2}=X_{1}^{-} v_{1}, \quad v_{3}=X_{2}^{-} v_{2}, \quad v_{4}=X_{2}^{-} v_{3} /[2]_{q}, \quad v_{5}=X_{1}^{-} v_{4}
$$

In this basis the action of the $X_{i}^{ \pm}$has matrix coefficients 0,1 or [2] $]_{q}$. We deform the action by replacing the $[2]_{q}$ by $[2]_{q t}$, that is to say we only deform $X_{2}^{ \pm} v_{3}=[2]_{q t} v_{3 \mp 1}$. The decomposition in simple modules for $\mathcal{U}_{1}$ and $\mathcal{U}_{2}$ is clear and coincides with the case $t=1$.

Example 2 Let $V=L\left(2 \omega_{2}\right)$ representation of $\mathcal{U}_{q}\left(B_{2}\right)$ of dimension 10 which corresponds by duality to the representation of $\mathcal{U}_{-t}\left(C_{2}\right)$ whose highest component is the 
fundamental representation of dimension 5. Its character is $y_{2}^{2}+y_{1}+y_{1}^{2} y_{2}^{-1}+y_{2}^{2} y_{1}^{-1}+$ $2.1+y_{2}^{2} y_{1}^{-2}+y_{1} y_{2}^{-1}+y_{1}^{-1}+y_{2}^{-2}$. There is a multiplicity 2 for the weight 1 .

We consider a basis $\left(v_{l}\right)_{1 \leq l \leq 10}$ of $V$ such that $v_{1}$ is a highest weight vector,

$$
\begin{aligned}
& v_{2}=X_{2}^{-} v_{1}, \quad v_{3}=X_{2}^{-} v_{2} /[2]_{q} \\
& v_{4}=X_{1}^{-} v_{3}, \quad v_{5}=X_{1}^{-} v_{4} /[2]_{q}, \quad v_{6}=X_{2}^{-} v_{5}, \\
& v_{7}=X_{2}^{-} v_{6} /[2]_{q}, \quad v_{8}=X_{1}^{-} v_{2}, \quad v_{9}=X_{2}^{-} v_{8}, \quad v_{10}=X_{2}^{-} v_{9} /[2]_{q} .
\end{aligned}
$$

In this basis the action of the $X_{i}^{ \pm}$have matrix coefficients $0,1,[2]_{q}$ or $[2]_{q^{2}}$. We deform the action by replacing these coefficients, respectively, by $0,1,[2]_{q t},[2]_{q^{2} t}$. That is to say we only deform

$$
X_{1}^{ \pm} v_{4}=[2]_{q^{2} t} v_{4 \mp 1}, \quad X_{2}^{ \pm} v_{6}=[2]_{q t} v_{6 \mp 1}, \quad X_{1}^{ \pm} v_{9}=[2]_{q t} v_{4 \mp 1}
$$

The decomposition in simple modules for $\mathcal{U}_{1}$ and $\mathcal{U}_{2}$ is clear and coincides with the case $t=1$ except for the trivial submodules of $\mathcal{U}_{2}$ and $\mathcal{U}_{1}$ which are, respectively,

$$
\mathbb{C}\left(v_{9}-[2]_{q t} v_{4}\right) \text { and } \mathbb{C}\left([2]_{q t} v_{4}-[2]_{q^{2} t} v_{9}\right)
$$

Note that a priori we cannot expect to have simple relations between the $C$ and the $X_{1}^{ \pm}$as $v_{4}$ is not an eigenvector of $C$.

Example 3 Let $V=L\left(2 \omega_{1}\right)$, an irreducible representation of $\mathcal{U}_{q}\left(B_{2}\right)$ of dimension 14 , which corresponds by duality to a representation of $\mathcal{U}_{-t}\left(C_{2}\right)$ whose highest component is of dimension 10 . Its character is $y_{1}^{2}+y_{2}^{2}+y_{2}^{4} y_{1}^{-2}+y_{1}+y_{2}^{2} y_{1}^{-1}+y_{1}^{2} y_{2}^{-2}+$ $2.1+y_{1} y_{2}^{-2}+y_{2}^{2} y_{1}^{-2}+y_{1}^{2} y_{2}^{-4}+y_{1}^{-1}+y_{2}^{-2}+y_{1}^{-2}$, and there is a multiplicity 2 for the weight 1 .

We consider a basis $\left(v_{l}\right)_{1 \leq l \leq 14}$ of $V$ such that $v_{1}$ is a highest weight vector,

$$
\begin{aligned}
v_{2} & =X_{1}^{-} v_{1}, \quad v_{3}=X_{1}^{-} v_{2} /[2]_{q^{2}}, \quad v_{4}=X_{2}^{-} v_{3}, \quad v_{5}=X_{2}^{-} v_{4} /[2]_{q}, \\
v_{6} & =X_{2}^{-} v_{5} /[3]_{q}, \quad v_{7}=X_{2}^{-} v_{6} /[4]_{q}, \quad v_{8}=X_{1}^{-} v_{7}, \quad v_{9}=X_{1}^{-} v_{8} /[2]_{q^{2}}, \\
v_{10} & =X_{2}^{-} v_{2}, \quad v_{11}=X_{2}^{-} v_{10} /[2]_{q}, \quad v_{12}=X_{1}^{-} v_{11}, \\
v_{13} & =X_{1}^{-} v_{12} /[2]_{q^{2}}, \quad v_{14}=X_{1}^{-} v_{6} .
\end{aligned}
$$

In this basis the action of the $X_{i}^{ \pm}$have matrix coefficients $0,1,[2]_{q},[3]_{q},[4]_{q},[2]_{q^{2}}$, $\left([2]_{q}[2]_{q^{2}} /[4]_{q}\right)^{ \pm 1},[4]_{q} /[2]_{q^{2}}$. We deform the action by replacing these coefficients, respectively, by $0,1,[2]_{q t},[3]_{q},[4]_{q t},[2]_{q^{2} t},\left([2]_{q t}[2]_{q^{2} t} /[4]_{q t}\right){ }^{ \pm 1},[4]_{q t} /[2]_{q^{2} t}$. The decomposition into simple modules for $\mathcal{U}_{1}$ and $\mathcal{U}_{2}$ is clear and coincides with the case $t=1$ except for the trivial submodule of $\mathcal{U}_{2}$ and $\mathcal{U}_{1}$ which are, respectively,

$$
\mathbb{C}\left([3]_{q}[4]_{q t} v_{12}-[2]_{q t}[2]_{q^{2} t} v_{5}\right) \text { and } \mathbb{C}\left([2]_{q t}[2]_{q^{2} t}^{2} v_{12}-[4]_{q t} v_{5}\right) .
$$


In this example we can observe non-trivial $t$-deformations of the Serre relations, but different relations are satisfied on different vectors in the representation! Indeed, we have

$$
\begin{aligned}
& \left(X_{2}^{-} X_{1}^{-2}-\left(q^{2} t^{2}+q^{-2} t^{-2}\right) X_{1}^{-} X_{2}^{-} X_{1}^{-}+X_{1}^{-2} X_{2}^{-}\right) v_{1}=0 \\
& \left(X_{2}^{-} X_{1}^{-2}-\left(q^{2} t+q^{-2} t^{-1}\right) X_{1}^{-} X_{2}^{-} X_{1}^{-}+X_{1}^{-2} X_{2}^{-}\right) v_{11}=0
\end{aligned}
$$

This implies that if we impose any $t$-deformation of the Serre relation

$$
\left(X_{2}^{-} X_{1}^{-2}-\left(q^{2}+q^{-2}\right) X_{1}^{-} X_{2}^{-} X_{1}^{-}+X_{1}^{-2} X_{2}^{-}\right)=0
$$

in the algebra $\mathcal{U}_{q, t}\left(B_{2}\right)$, then the resulting algebra will not act on the $\mathcal{U}_{q, t}\left(B_{2}\right)$-module that we have just constructed. Indeed, this relation will be different from the relation satisfied on at least one of the vectors, $v_{1}$ and $v_{11}$ (as written above). The difference of the two relations would give us a multiple of the monomial $X_{1}^{-} X_{2}^{-} X_{1}^{-}$, which should then have to annihilate this vector. But neither vector is annihilated by this monomial: we have

$$
\begin{aligned}
\left(X_{1}^{-} X_{2}^{-} X_{1}^{-}\right) v_{1} & =v_{4}[2]_{q t}[2]_{q^{2} t} /[4]_{q t} \\
\left(X_{1}^{-} X_{2}^{-} X_{1}^{-}\right) v_{11} & =v_{14}[2]_{q t}[2]_{q^{2} t} /[4]_{q t} .
\end{aligned}
$$

Moreover, one can show that the structure of $\mathcal{U}_{q, t}(\mathfrak{g})$-module on $V$ described above is unique (the same is also true for the modules in Examples 1 and 2).

Now let us explain how we constructed the above interpolating representations. Let $V$ be a simple finite-dimensional representation of $\mathcal{U}_{q}\left(B_{2}\right)$ as above of highest weight $\lambda=m_{1} \omega_{1}+2 m_{2} \omega_{2}$. We have a decomposition in weight spaces $V=\bigoplus_{\mu \leq \lambda} V_{\mu}$. Let $V_{r}=\bigoplus_{\mu=\lambda-\alpha_{i_{1}}-\cdots-\alpha_{i_{r}}} V_{\mu}$ and $V_{\leq R}=\bigoplus_{r \leq R} V_{r}$. We have $X_{1}^{-} V_{R}+X_{2}^{-} V_{R}=V_{R+1}$ and $V_{\lambda}=X_{1}^{-} V_{\lambda-\alpha_{1}}+X_{2}^{-} V_{\lambda-\alpha_{2}}$.

We define on $V$ the action of the $K_{i}(t), \tilde{K}_{i}(t)$ as explained above.

We define by induction on $r \geq 0$ the deformed actions

$$
X_{1}^{+}(t), X_{2}^{+}(t): V_{r+1} \rightarrow V_{r} \quad \text { and } \quad X_{1}^{-}(t), X_{2}^{-}(t): V_{r} \rightarrow V_{r+1}
$$

satisfying the following properties:

(i) $X_{1}^{+}(1)=X_{1}^{+}, X_{2}^{+}(1)=X_{2}^{+}, X_{1}^{-}(1)=X_{1}^{-}, X_{2}^{-}(1)=X_{2}^{-}$,

(ii) the action is compatible with the restrictions to $\mathcal{U}_{1}$ and $\mathcal{U}_{2}$,

(iii) $\left[X_{1}^{+}(t), X_{2}^{-}(t)\right]=0$,

(iv) $\left[X_{2}^{+}(t), X_{1}^{-}(t)\right]=0$.

To start with we set $X_{1}^{+}(t)=0, X_{2}^{+}(t)=0$ on $V_{0}$. 
Suppose that the deformed action is defined for $r \geq 0$. Let $V_{\lambda} \subset V_{r+1}$. We want to define the deformed actions

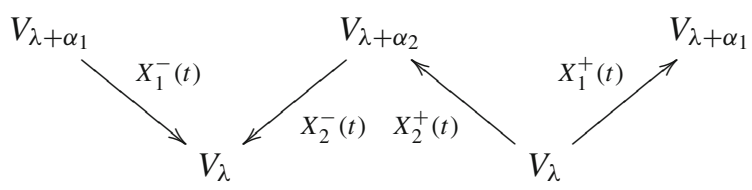

By using the condition (ii) for $\mathcal{U}_{2}$, we can first define the action of $X_{2}^{+}(t)$ and $X_{2}^{-}(t)$. This gives in particular a decomposition $V_{\lambda}=V_{\lambda}^{(2)} \oplus \tilde{V}_{\lambda}^{(2)}$ where $V_{\lambda}^{(2)}=$ $X_{2}^{-}(t)\left(V_{\lambda+\alpha_{2}}\right)$ and $\tilde{V}_{\lambda}^{(2)}=\operatorname{Ker}\left(X_{2}^{+}(t)\right) \cap V_{\lambda}$. The condition (iii) on $V_{\lambda+\alpha_{2}}$ gives $\phi(t): V_{\lambda}^{(2)} \rightarrow V_{\lambda+\alpha_{1}}$. The condition (iv) on $V_{\lambda+\alpha_{1}}$ gives $\psi(t): V_{\lambda+\alpha_{1}} \rightarrow V_{\lambda}^{(2)}$. So it suffices to define $X_{1}^{ \pm}(t)$ such that $X_{1}^{+}(t)=\phi(t)$ on $V_{\lambda}^{(2)}, \Pi \circ X_{1}^{-}(t)=\Psi(t)$ where $\Pi$ is the projection on $V_{\lambda}^{(2)}$ along $\tilde{V}_{\lambda}^{(2)}$, and $X_{1}^{+}(t) X_{1}^{-}(t)=R(t)$ given by condition (ii) for $\mathcal{U}_{1}$. In a matrix form we have $X_{1}^{+}(t)=(\phi(t) A(t)), X_{1}^{-}(t)=\left(\begin{array}{l}\Psi(t) \\ B(t)\end{array}\right)$, $X_{1}^{-}(t) X_{1}^{+}(t)=\phi(t) \psi(t)+A(t) B(t)$.

So it suffices to prove that

$$
\operatorname{rk}(R(t)-\phi(t) \psi(t)) \leq \operatorname{dim}\left(\tilde{V}_{\lambda}^{(2)}\right)
$$

We call this the compatibility condition. In the examples studied above this condition is satisfied, and that is why the interpolating representations do exist. We conjecture that it is satisfied in general and we have the following

Conjecture 1 For any $\lambda \in P^{\prime}$ there exists a unique irreducible representation $L_{q, t}(\lambda)$ of $\mathcal{U}_{q, t}(\mathfrak{g})$ whose specialization at $t=1$, viewed as a $\mathcal{U}_{q}(g)$-module, is the irreducible module $L(\lambda)$ and specialization at $q=\epsilon$, viewed as a $\mathcal{U}_{-t}\left({ }^{L} \mathfrak{g}\right)$-module, contains a module of character $\Pi(\chi(\lambda))$.

\section{Conjectures on the Langlands duality for quantum groups}

In this section we conjecture stronger statements on the duality for characters and crystals which we prove for simply laced $\mathfrak{g}$ with $r=2$ and for $B_{2}$. The proof of these conjectures and the computation of the corresponding Langlands duality branching rules is a program for further study for this Langlands duality.

\subsection{A positivity conjecture}

It is easy to compute the Langlands duality branching rules for the examples of Sect. 2.1. 
For $\mathfrak{g}=B_{2}$ :

$$
\begin{aligned}
\Pi\left(\chi\left(\omega_{1}\right)\right) & =\chi^{L}\left(\check{\omega}_{1}\right)+\chi^{L}(0), \quad \Pi\left(\chi\left(2 \omega_{2}\right)\right)=\chi^{L}\left(\check{\omega}_{2}\right)+\chi^{L}\left(\check{\omega}_{1}\right)+\chi^{L}(0), \\
\Pi\left(\chi\left(2 \omega_{1}\right)\right) & =\chi^{L}\left(2 \check{\omega}_{1}\right)+\chi^{L}\left(\check{\omega}_{1}\right), \\
\Pi\left(\chi\left(\omega_{1}+2 \omega_{2}\right)\right) & =\chi^{L}\left(\check{\omega}_{1}+\check{\omega}_{2}\right)+\chi^{L}\left(2 \check{\omega}_{1}\right)+\chi^{L}\left(2 \check{\omega}_{2}\right)+\chi^{L}\left(\check{\omega}_{1}\right) .
\end{aligned}
$$

and for $\mathfrak{g}=G_{2}$ :

$$
\Pi\left(\chi\left(\omega_{1}\right)\right)=\chi^{L}\left(\check{\omega}_{1}\right)+\chi^{L}(0), \Pi\left(\chi\left(\omega_{2}\right)\right)=\chi^{L}\left(\check{\omega}_{2}\right)+2 \chi^{L}\left(\check{\omega}_{1}\right)+\chi^{L}(0) .
$$

So it is natural to give a purely classical analog to Conjecture 1:

Conjecture 2 For any $\lambda \in P^{+} \cap P^{\prime}, \Pi(\chi(\lambda))$ is the character of an ${ }^{L} \mathfrak{g}$-module.

This Conjecture means that the virtual representation of Proposition 1 is an actual representation, that is, the Langlands duality branching rules are positive:

$$
\Pi(\chi(\lambda))=\sum_{\check{\mu} \in P^{L,+}} m_{\check{\mu}} \chi^{L}(\check{\mu}), \quad m_{\check{\mu}} \in \mathbb{Z}_{+} .
$$

We will prove the conjecture in several cases, but first we prove that in general certain Langlands duality branching rules are positive. We use the partial ordering on $P^{\prime}$ viewed as the ${ }^{L} \mathfrak{g}$ weight lattice.

Proposition 6 Let $\check{\mu}_{0}$ maximal in $\left\{\check{\mu} \in P^{L,+} \mid m_{\breve{\mu}} \neq 0, \check{\mu} \neq \Pi(\lambda)\right\}$. Then $m_{\check{\mu}_{0}}>0$.

Proof By Theorem 1 the coefficient of $\check{\mu}_{0}$ in $\Pi(\chi(\lambda))$ is larger than in $\chi^{L}(\Pi(\lambda))$. But the only terms which can contribute to this multiplicity are by hypothesis $\chi^{L}(\Pi(\lambda))$ and $\chi^{L}\left(\check{\mu}_{0}\right)$. This implies the result.

This includes all coefficients in the examples at the beginning of this section. Now let us consider a statement analogous to Conjecture 2 in terms of crystals.

\subsection{Structure of the crystal $\tilde{\mathcal{B}}(\lambda)$}

For $\lambda \in P^{+} \cap P^{\prime}$ let

$$
\tilde{\mathcal{B}}(\lambda)=\left\{v \in \mathcal{B}(\lambda) \mid \operatorname{wt}\left(m^{\prime}\right) \in P^{\prime}\right\}
$$

Note that for $\mathfrak{g}$ of type $B_{\ell}$ we have $\tilde{\mathcal{B}}(\lambda)=\mathcal{B}(\lambda)$.

$\tilde{\mathcal{B}}(\lambda) \sqcup\{0\}$ is stable under the action of the operators $e_{i}^{L}, f_{i}^{L}$. We define new maps $\epsilon_{i}^{L}, \phi_{i}^{L}$ on $\tilde{\mathcal{B}}(\lambda)$ corresponding to the operators $e_{i}^{L}, f_{i}^{L}$ (in general they do not coincide with the original $\epsilon_{i}, \phi_{i}$, as we may have $e_{i}^{L}\left(m^{\prime}\right)=0$ but $e_{i}^{L}\left(m^{\prime}\right) \neq 0$, see the example in Sect. 2.4). We get an abstract ${ }^{L} \mathfrak{g}$-crystal $\left(\tilde{\mathcal{B}}(\lambda), e_{i}^{L}, f_{i}^{L}, \epsilon_{i}^{L}, \phi_{i}^{L}\right.$, wt $\left.{ }^{L}\right)$. Moreover, this crystal is semi-normal, that is to say that for each $i \in I$, it is as a ${ }^{L} \mathfrak{g}_{\{i\}}$-crystal 
the crystal of a ${ }^{L} \mathfrak{g}_{\{i\}}$-module (this is a direct consequence of the semi-normal property of $\mathcal{B}(\lambda)$ ). Here for $J \subset I$ we denote by $\mathfrak{g}_{J}$ the sub Lie algebra of Cartan matrix $\left(C_{i, j}\right)_{i, j \in J}$.

Theorem 2 means that the connected component of $\tilde{\mathcal{B}}(\lambda)$ containing the highest weight vector is normal, that it to say is the crystal of a ${ }^{L} \mathfrak{g}$-module. In this section we study the structure of the whole crystal $\tilde{\mathcal{B}}(\lambda)$.

In all examples of Sect. 2.4 the crystal is normal. In particular, we have obtained the following:

Proposition 7 For all pseudo fundamental representations of a rank 2 Lie algebra, $\tilde{\mathcal{B}}(\lambda)$ is normal.

So we could expect naively that $\tilde{\mathcal{B}}(\lambda)$ is normal. This statement is not true in general. For example in type $B_{2}$ consider $\lambda=\omega_{1}+2 \omega_{2}$.

We have seen that in terms of characters $\Pi\left(\chi\left(\omega_{1}+2 \omega_{2}\right)\right)$ has 4 simple constituents. But $\mathcal{B}(\lambda)=\mathcal{M}\left(Y_{1,0} Y_{2,1}^{2}\right)=\tilde{\mathcal{B}}(\lambda)$ has 3 connected component as a ${ }^{L} \mathfrak{g}$-crystal.

The first connected component is isomorphic to $\mathcal{B}^{L}\left(\check{\omega}_{1}+\check{\omega}_{2}\right)(16$ terms): $\left\{1_{0} 2_{1}^{2}, 1_{2}^{-1} 2_{1}^{4}, 1_{0} 1_{2}^{2} 2_{3}^{-2}, 2_{1}^{2} 2_{3}^{-2} 1_{2}, 1_{0} 1_{2} 1_{4}^{-1}, 2_{1}^{2} 1_{4}^{-1}, 1_{0} 1_{4}^{-2} 2_{3}^{2}, 2_{3}^{-4} 1_{2}^{3}\right.$, $\left.1_{2}^{-1} 1_{4}^{-2} 2_{1}^{2} 2_{3}^{2}, 2_{3}^{-2} 1_{2}^{2} 1_{4}^{-1}, 1_{0} 2_{5}^{-2}, 1_{2} 1_{4}^{-2}, 1_{2}^{-1} 2_{1}^{2} 2_{5}^{-2}, 1_{4}^{-3} 2_{3}^{2}, 2_{3}^{-2} 2_{5}^{-2} 1_{2}, 1_{4}^{-1} 2_{5}^{-2}\right\}$.

The second connected component is isomorphic to $\mathcal{B}^{L}\left(\omega_{1}\right)$ (4 terms): $\left\{1_{0} 2_{1} 2_{5}^{-1}, 1_{2}^{-1} 2_{1}^{3} 2_{5}^{-1}, 2_{1} 2_{3}^{-2} 2_{5}^{-1} 1_{2}, 1_{4}^{-1} 2_{1} 2_{5}^{-1}\right\}$.

The third connected component is (15 terms):

$\left\{1_{0} 1_{2} 2_{1} 2_{3}^{-1}, 2_{1}^{3} 2_{3}^{-1}, 1_{0} 1_{4}^{-1} 2_{1} 2_{3}, 1_{2}^{-1} 1_{4}^{-1} 2_{1}^{3} 2_{3}, 2_{1} 2_{3}^{-3} 1_{2}^{2}, 1_{0} 1_{2} 2_{3}^{-1} 2_{5}^{-1}\right.$, $2_{1} 2_{3}^{-1} 1_{2} 1_{4}^{-1}, 2_{1}^{2} 2_{3}^{-1} 2_{5}^{-1}, 1_{0} 1_{4}^{-1} 2_{3} 2_{5}^{-1}, 1_{2}^{-1} 1_{4}^{-1} 2_{1}^{2} 2_{3} 2_{5}^{-1}, 1_{4}^{-2} 2_{1} 2_{3}, 1_{2}^{2} 2_{3}^{-3} 2_{5}^{-1}$, $\left.2_{1} 2_{3}^{-1} 2_{5}^{-2}, 1_{2} 1_{4}^{-1} 2_{3}^{-1} 2_{5}^{-1}, 1_{4}^{-2} 2_{3} 2_{5}^{-1}\right\}$.

Although the third component has two highest weight elements $u=1_{0} 1_{2} 2_{1} 2_{3}^{-1}$ and $v=2{ }_{1}^{3} 2_{3}^{-1}$ it is not connected as

$$
e_{2}^{L} e_{1}^{L} e_{1}^{L} e_{2}^{L} v=1_{2} 1_{4}^{-1} 2_{3}^{-1} 2_{5}^{-1}=e_{1}^{L} e_{2}^{L} e_{2}^{L} e_{1}^{L} e_{1}^{L} u
$$

But its character is $\chi^{L}\left(\check{\omega}_{1}\right)+\chi^{L}\left(\check{\omega}_{2}\right)$.

In fact, it suffices to modify slightly the crystal structure of the third component to get a normal crystal. Indeed, without changing the $\mathrm{wt}^{L}, \epsilon_{i}^{L}, \phi_{i}^{L}$, we just replace

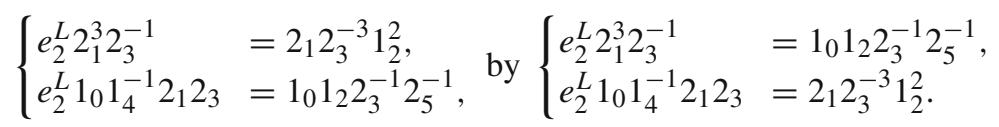

In other words we have defined a bijection $\Psi: \tilde{\mathcal{B}}(\lambda) \rightarrow \mathcal{B}^{L}$, where $\mathcal{B}^{L}$ is normal, satisfying $\left(\mathrm{wt}^{L}, \phi_{i}^{L}, \epsilon_{i}^{L}\right) \Psi=\left(\mathrm{wt}^{L}, \phi_{i}^{L}, \epsilon_{i}^{L}\right)$ and $\Psi f_{1}^{L}=f_{1}^{L} \Psi, \Psi e_{1}^{L}=e_{1}^{L} \Psi$. But $\Psi$ is not a morphism of crystal as $\Psi f_{2}^{L} \neq f_{2}^{L} \Psi$ and $\Psi e_{2}^{L} \neq e_{2}^{L} \Psi$ (see the picture bellow). 


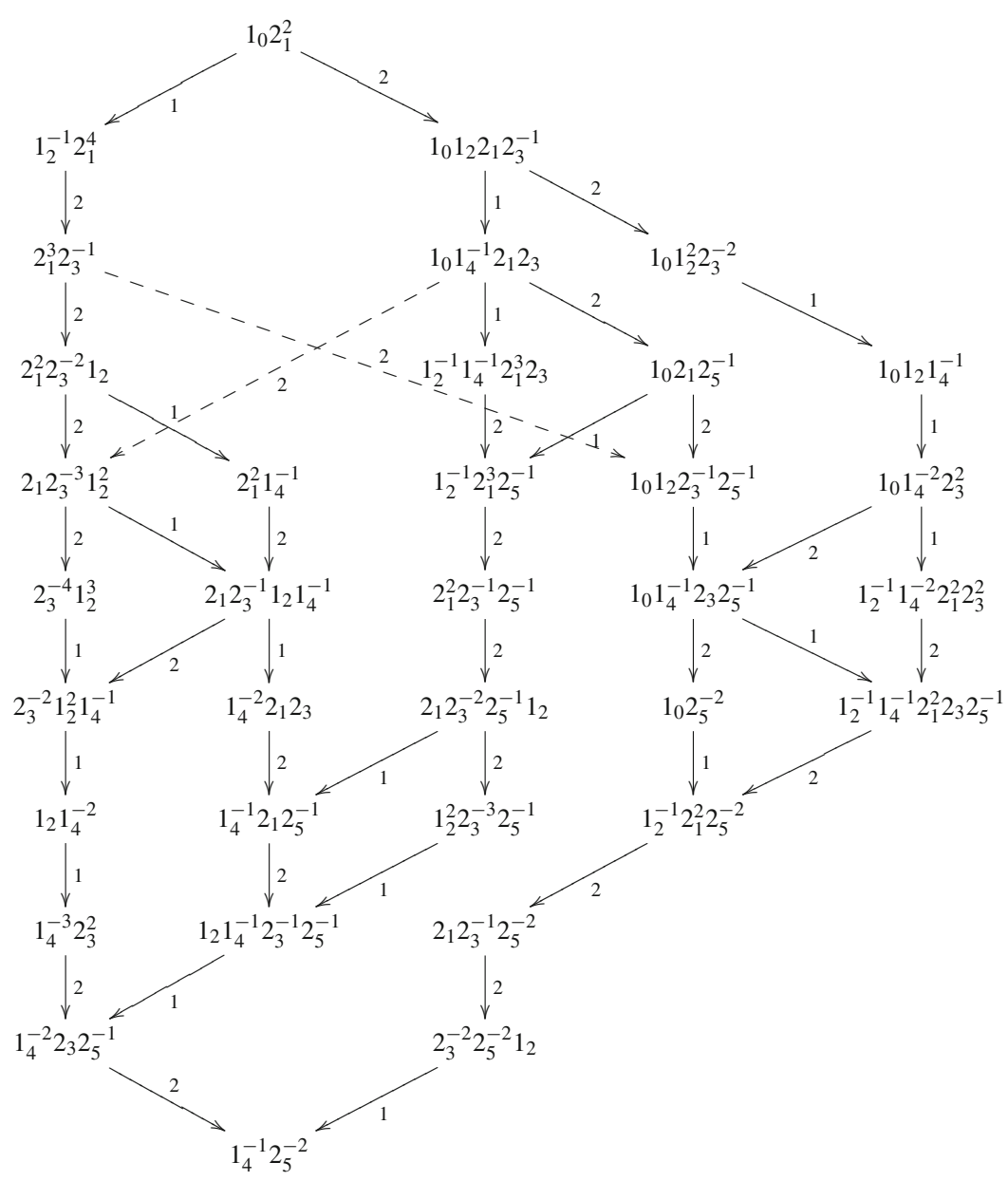

Conjecture 3 For $\lambda \in P^{\prime}$, there is a bijection $\Psi: \tilde{\mathcal{B}}(\lambda) \rightarrow \mathcal{B}^{L}$ to a normal ${ }^{L} \mathfrak{g}$-crystal $\mathcal{B}^{L}$ satisfying $\mathrm{wt}^{L} \Psi=\mathrm{wt}^{L}$ and $\left(\phi_{i}^{L}, \epsilon_{i}^{L}\right) \Psi=\left(\phi_{i}^{L}, \epsilon_{i}^{L}\right)$ for any $i \in I$.

This means that, by changing the maps $e_{i}^{L}, f_{i}^{L}$, respectively, by $\Psi^{-1} e_{i}^{L} \Psi, \Psi^{-1} f_{i}^{L} \Psi$, we get a normal crystal.

Conjecture 3 implies Conjecture 2 as we have

$$
\Pi(\chi(\lambda))=\sum_{v \in \tilde{\mathcal{B}}(\lambda)} \mathrm{wt}^{L}(v) .
$$

First, we look at the case of the Lie algebra of rank 1. For $r=1$ the result is clear as $e^{L}=e$ and $f^{L}=f$. For $r=2$, consider $\mathcal{B}(2 p \omega)=\tilde{\mathcal{B}}(2 p \omega)$ :

$$
u_{2 p} \rightarrow u_{2(p-1)} \rightarrow u_{2(p-2)} \rightarrow \cdots \rightarrow u_{2(2-p)} \rightarrow u_{2(1-p)} \rightarrow u_{-2 p}
$$

which is decomposed in $\mathcal{B}^{L}(p \check{\omega}) \sqcup \mathcal{B}^{L}((p-1) \check{\omega})$ as a ${ }^{L} \mathfrak{g}$-crystal:

$$
\left(u_{2 p} \rightarrow u_{2(p-2)} \rightarrow \cdots \rightarrow u_{-2 p}\right) \sqcup\left(u_{2 p-2} \rightarrow u_{2 p-6} \rightarrow \cdots \rightarrow u_{2-2 p}\right) .
$$


Here Conjecture 2 is just the elementary decomposition:

$$
\begin{aligned}
& y^{2 p}+y^{2 p-2}+\cdots+y^{-2 p} \\
& \quad=\left(y^{2 p}+y^{2 p-4}+\cdots+y^{-2 p}\right)+\left(y^{2 p-2}+y^{2 p-6}+\cdots+y^{2-2 p}\right) .
\end{aligned}
$$

We have the following Theorem, due to [20,21] (see for example [19, Theorem 2.1]):

Theorem 5 A finite $\mathfrak{g}$-crystal $B$ is normal if and only if for any $J \subset I$ with at most two elements, $B$ is normal as a $\mathfrak{g}_{J}$-crystal.

So it is of particular importance to study Lie algebras of rank 2. We will now prove Conjecture 3 for Lie algebras of rank 2 (and $r \leq 2$ ). Such a Lie algebra will be denoted by $\left(X, r_{1}, r_{2}\right)$, where $1 \leq r_{1}, r_{2} \leq 2$ are the labels. We consider all crystals $\mathcal{B}(\lambda)$ such that $\tilde{\mathcal{B}}(\lambda) \neq \emptyset$. For $\mathfrak{g}$ of type $B_{2}$ it implies $\lambda \in P^{\prime}$ but in general $\lambda$ is not necessarily in $P^{\prime}$. For $\left(A_{1} \times A_{1}, 2,2\right)$ and $\left(A_{2}, 2,2\right)$ the result is clear as we have $f_{i}^{L}=f_{i}$ and $e_{i}^{L}=e_{i}$. For types $\left(A_{1} \times A_{1}, 1,1\right),\left(A_{1} \times A_{1}, 1,2\right),\left(A_{1} \times A_{1}, 2,1\right)$, the result follows from the rank 1 -case studied above. So we study the two remaining case $\left(A_{2}, 1,1\right)$ and $\left(B_{2}, 1,2\right)$. In fact, we prove

Theorem 6 Conjectures 2 and 3 hold for simply laced $\mathfrak{g}$ with $r=2$ and for $B_{2}$.

We cannot prove the statement for $B_{2}$ directly by using the result for pseudofundamental representations (Proposition 7) as the $e_{i}^{L}, f_{i}^{L}$ for the tensor product of ${ }^{L} \mathfrak{g}$-crystals do not coincide with the operators defined from the tensor product of $\mathfrak{g}$-crystals.

\subsection{Type $\left(A_{2}, 1,1\right)$}

Let $\lambda=R \omega_{1}+R^{\prime} \omega_{2}$ dominant in $P$. We have $\lambda \equiv 0, \omega_{1}, \omega_{2}$ or $\omega_{1}+\omega_{2}$ where $\equiv$ means mod $P^{\prime}$ in this section. Let $\Lambda=\{(i, 1) \mid 1 \leq i \leq R\} \cup\{(i, j) \mid R+1 \leq$ $\left.i \leq R+R^{\prime}, 1 \leq j \leq 2\right\}$. Then $\mathcal{B}(\lambda)$ is isomorphic [22] to the crystal of tableaux $\left(T_{i, j}\right)_{(i, j) \in \Lambda}$ with coefficients in $\{1,2,3\}$ which are semi-standard (i.e., $T_{i, j} \leq T_{i+1, j}$ and any $i, j$, and $T_{i, 1}>T_{i, 2}$ for $\left.i \geq 2 R+1\right)$. Let

$$
T_{\lambda}=\left(\begin{array}{llllll} 
& & & 1 & \cdots & 1 \\
1 & \cdots & 1 & 2 & \cdots & 2
\end{array}\right)
$$


be the highest weight tableaux. Let us compute the tableaux $T \in \tilde{\mathcal{B}}(\lambda)$ of highest weight for $e_{1}^{L}=e_{1}^{2}$ and $e_{2}^{L}=e_{2}^{2} . T=T(a, b, c)$ is characterized by $a, b, c$ such that $T_{i, 1}=\left\{\begin{array}{ll}1 & \text { for } i \leq a-1, \\ 2 & \text { for } a \leq i \leq b-1, \\ 3 & \text { for } b \leq i,\end{array} \quad\right.$ and $\quad T_{i, 2}= \begin{cases}1 & \text { for } R+1 \leq i \leq c-1, \\ 2 & \text { for } c \geq i\end{cases}$

The condition $e_{1}^{2} T=0$ is equivalent to the following:

$$
\left(R+R^{\prime}=c \text { and } b-a \leq R^{\prime}-1\right) \quad \text { or } \quad\left(R+R^{\prime}=c-1 \text { and } b-a \leq R^{\prime}+1\right) .
$$

The condition $e_{2}^{2} T=0$ is equivalent to $c-b \in\{0,1\}$. We have four cases:

(1) $c=b=R+R^{\prime}$ and $a \geq R+1$. So $a=R+1$. So $0 \equiv \operatorname{wt}(T) \equiv \lambda+\omega_{1}+\omega_{2}$.

(2) $c=b=R+R^{\prime}+1$ and $a \geq R$. If $a=R+1$ then $T=T_{\lambda}$ and $\lambda \equiv 0$. If $a=R$, then $\lambda \equiv \omega_{2}$.

(3) $c=b+1=R+R^{\prime}$ and $a \geq R$. If $a=R+1$, then $\lambda \equiv \omega_{2}$. If $a=R$, then $\lambda \equiv 0$.

(4) $c=b+1=R+R^{\prime}+1$ and $a \geq R-1$. If $a=R+1$ then $\lambda \equiv \omega_{1}$. If $a=R$ then $\lambda \equiv \omega_{1}+\omega_{2}$. If $a=R-1$ then $\lambda \equiv \omega_{1}$.

So for each value of $\lambda \bmod P^{\prime}$ we have 2 highest weight vectors $T_{0}, T_{1}$ of respective connected component $\mathcal{B}^{\prime}$ and $\mathcal{B}^{\prime \prime}$. We prove that $\mathcal{B}^{\prime} \neq \mathcal{B}^{\prime \prime}$ and that they are normal. This implies a stronger result than Conjecture 3 , that in this case $\tilde{B}(\lambda)$ is normal.

$$
\left(T_{0}, T_{1}\right)= \begin{cases}\left(T_{\lambda}, f_{1} f_{2} f_{1} f_{2} T_{\lambda}\right) & \text { if } \lambda \equiv 0 \\ \left(f_{2} T_{\lambda}, f_{2} f_{1} f_{1} T_{\lambda}\right) & \text { if } \lambda \equiv \omega_{1} \\ \left(f_{1} T_{\lambda}, f_{1} f_{2} f_{2} T_{\lambda}\right) & \text { if } \lambda \equiv \omega_{2} \\ \left(f_{1} f_{2} T_{\lambda}, f_{2} f_{1} T_{\lambda}\right) & \text { if } \lambda \equiv \omega_{1}+\omega_{2}\end{cases}
$$

Note that $\operatorname{wt}(T(a, b, c)) \in P^{\prime}$ if and only if $b \equiv 1+R^{\prime}[2]$ and $a \equiv c[2]$.

Let us treat in detail the case $\lambda \equiv 0$. We know by Theorem 2 that $\mathcal{B}^{\prime}$ is normal. In particular, $\mathcal{B}^{\prime} \neq \mathcal{B}^{\prime \prime}$. So we only have to prove that $\mathcal{B}^{\prime \prime}(\lambda)$ is isomorphic as a ${ }^{L} \mathfrak{g}$-crystal to $\mathcal{B}^{L}\left(\lambda^{\prime}\right)$ where $\lambda^{\prime}=\Pi\left(\mathrm{wt}\left(T_{1}\right)\right)=(R / 2-1) \omega_{1}+\left(R^{\prime} / 2-1\right) \omega_{2}$. We have $T_{1}=T\left(R, R+R^{\prime}-1, R+R^{\prime}\right)$ that is,

$$
T_{1}=\left(\begin{array}{lllllllll} 
& & 1 & \cdots & 1 & 1 & 2 \\
1 & \cdots & 1 & 2 & 2 & \cdots & 2 & 3 & 3
\end{array}\right) .
$$

Let $\Lambda^{\prime}=\{(i, 1) \mid 1 \leq i \leq R / 2-1\} \cup\left\{(i, j) \mid R / 2+1 \leq i \leq R / 2+R^{\prime} / 2-1,1 \leq j \leq 2\right\}$. Then $\mathcal{B}^{L}\left(\lambda^{\prime}\right)$ is isomorphic to the crystal of semi-standard tableaux $\left(T_{i, j}\right)_{(i, j) \in \Lambda^{\prime}}$ with coefficients in $\{1,2,3\}$. For such a tableaux we define $a, b, c$ as above. Then consider

$$
\phi: T(a, b, c) \in \mathcal{B}^{L}\left(\lambda^{\prime}\right) \rightarrow T(2 a, 2 b+1,2 c+2) \mathcal{B}^{\prime \prime}(\lambda) .
$$


Then $\phi$ is an isomorphism of ${ }^{L} \mathfrak{g}$-crystals. First for $\left(R / 2,\left(R+R^{\prime}\right) / 2-1, R / 2+\right.$ $\left.R^{\prime} / 2-1\right)$ we get $T\left(R, R+R-1, R+R^{\prime}\right)=T_{1}$. Then it suffices to prove that $\phi\left(f_{i} T\right)=f_{i}^{2} \phi(T)$. Let $T=T(a, b, c)$.

For $f_{1}$ : if $R+b \geq a+c$ and $a \geq 2$, then $f_{1} T=T(a-1, b, c)$. We have $(R+1)+(2 b+1) \geq 2 a+(2 c+2)$, so $f_{1} \phi(T)=T(2 a-1,2 b+1,2 c+2)$. But we have also $(R+1)+(2 b+1) \geq(2 a-1)+(2 c+2)$ so $f_{1}^{2} \phi(T)=T(2(a-1), 2 b+1,2 c+2)$.

If $R+b \geq a+c$ and $a=1$, then $f_{1} T=0$. We have $(2 R+1)+(2 b+1) \geq$ $2 a+(2 c+2)$, so $f_{1} \phi(T)=T(1,2 b+1,2 c+2)$. But we have also $(R+1)+(2 b+1) \geq$ $(2 a-1)+(2 c+2)$ so $f_{1}^{2} \phi(T)=0$.

If $R+b<a+c$ and $c>b$ then $f_{1} T$ corresponds to $(a, b, c-1)$. We have $(2 R+1)+(2 b+1) \leq 2 a+2 c<2 a+2 c+2$, so $f_{1} \phi(T)=T(2 a, 2 b+1,2 c+1)$. But we have also $(R+1)+(2 b+1)<2 a+(2 c+1)$ so $f_{1}^{2} \phi(T)=T(2 a, 2 b+1,2(c-1)+2)$.

If $R+b<a+c$ and $c=b$ then $f_{1} T=0$. We have $(2 R+1)+(2 b+1) \leq$ $2 a+2 c<2 a+2 c+2$, so $f_{1} \phi(T)=T(2 a, 2 b+1,2 c+1)$. But we have also $(R+1)+(2 b+1)<2 a+(2 c+1)$ so $f_{1}^{2} \phi(T)=0$.

For $f_{2}$ : if $b>a$ then $f_{2} T=T(a, b-1, c)$. We have $2 b+1 \geq 2 a+3>2 a$, so $f_{1} \phi(T)=T(2 a, 2 b, 2 c+2)$. But we have also $2 b>2 a$, so $f_{1}^{2} \phi(T)=T(2 a, 2 b-1$, $2 c+2)$.

If $b=a$ then $f_{2} T=0$. We have $2 b+1=2 a+1>2 a$, so $f_{1} \phi(T)=$ $T(2 a, 2 b, 2 c+2)$. But then $2 b=2 a$, so $f_{1}^{2} \phi(T)=0$.

For the cases $\lambda \equiv \omega_{1}$ or $\lambda \equiv \omega_{1}+\omega_{2}$ we give only the formulas of isomorphisms of ${ }^{L} \mathfrak{g}$-crystals as above.

Let $\lambda \equiv \omega_{1}$ (the case $\lambda \equiv \omega_{2}$ is symmetric). $T_{0}=T\left(R+1, R+R^{\prime}, R+R^{\prime}+1\right)$, $T_{1}=T\left(R-1, R+R^{\prime}, R+R^{\prime}+1\right)$. Let $\phi^{\prime}: \mathcal{B}\left((R+1) / 2 \omega_{1}+\left(R^{\prime} / 2-1\right) \omega_{2}\right) \rightarrow \mathcal{B}^{\prime}$

$$
\phi^{\prime}: T(a, b, c) \mapsto \begin{cases}T(R+1,2 b-1,2 c) & \text { if } a=(R+3) / 2 \\ T(2 a-1,2 b-1,2 c-1) & \text { if } a<(R+3) / 2\end{cases}
$$

and

$$
\phi^{\prime \prime}: T(a, b, c) \in \mathcal{B}\left((R-1) / 2 \omega_{1}+R^{\prime} / 2 \omega_{2}\right) \mapsto T(2 a, 2 b+1,2(c+1)) \in \mathcal{B}^{\prime \prime}
$$

For $\lambda \equiv \omega_{1}+\omega_{2}, T_{0}=T\left(R+1, R+R^{\prime}, R+R^{\prime}\right), T_{1}=T\left(R, R+R^{\prime}, R+R^{\prime}+1\right)$. Let

$$
\begin{aligned}
& \phi^{\prime}: T(a, b, c) \in \mathcal{B}\left((R-1) / 2 \omega_{1}+\left(R^{\prime}-1\right) / 2 \omega_{2}\right) \mapsto T(2 a, 2 b, 2 c) \in \mathcal{B}^{\prime}, \\
& \phi^{\prime \prime}: T(a, b, c) \in \mathcal{B}\left((R-1) / 2 \omega_{1}+\left(\left(R^{\prime}-1\right) / 2 \omega_{2}\right) \mapsto T(2 a-1,2 b, 2 c+1) \in \mathcal{B}^{\prime \prime} .\right.
\end{aligned}
$$

Remark 7 In the course of the proof we have found the following Langlands duality branching rules (see the end of Sect. 2.1) for irreducible representations of $\left(A_{2}, 1,1\right)$ 
and the symmetric ones: $\left(\lambda_{1}, \lambda_{2}>0\right)$

$$
\begin{aligned}
& \Pi\left(\chi\left(2 \lambda_{1} \omega_{1}+2 \lambda_{2} \omega_{2}\right)\right)=\chi^{L}\left(\lambda_{1} \check{\omega}_{1}+\lambda_{2} \check{\omega}_{2}\right)+\chi^{L}\left(\left(\lambda_{1}-1\right) \check{\omega}_{1}+\left(\lambda_{2}-1\right) \check{\omega}_{2}\right), \\
& \Pi\left(\chi\left(2 \lambda_{1} \omega_{1}\right)\right)=\chi^{L}\left(\lambda_{1} \check{\omega}_{1}\right), \Pi\left(\chi\left(\left(2 \lambda_{1}-1\right) \omega_{1}\right)\right)=\chi^{L}\left(\left(\lambda_{1}-1\right) \check{\omega}_{1}\right), \\
& \Pi\left(\chi\left(\left(2 \lambda_{1}+1\right) \omega_{1}+2 \lambda_{2} \omega_{2}\right)\right) \\
& \quad=\chi^{L}\left(\left(\lambda_{1}+1\right) \check{\omega}_{1}+\left(\lambda_{2}-1\right) \check{\omega}_{2}\right)+\chi^{L}\left(\left(\lambda_{1}-1\right) \check{\omega}_{1}+\lambda_{2} \check{\omega}_{2}\right), \\
& \Pi\left(\chi\left(\left(2 \lambda_{1}-1\right) \omega_{1}+2\left(\lambda_{2}-1\right) \omega_{2}\right)\right)=2 \chi^{L}\left(\left(\lambda_{1}-1\right) \check{\omega}_{1}+\left(\lambda_{2}-1\right) \check{\omega}_{2}\right) .
\end{aligned}
$$

\subsection{Application to symmetric cases}

Consider a simply laced $\mathfrak{g}$ with $r=2$.

Proposition 8 For $\lambda \in P^{\prime}, \tilde{\mathcal{B}}(\lambda)$ is normal.

In particular, Conjectures 2 and 3 hold for these types. In fact, we have proved a stronger result as the crystal is normal.

Proof By Theorem 5, it suffices to prove the result for the subalgebras of rank 2. For subalgebras of type $A_{2}$, the statement follows from Sect. 6.3. For the subalgebras of type $A_{1} \times A_{1}$, it suffices to prove that if $C_{i, j}=0$ then the $f_{i}^{L}, f_{j}^{L}$ commute. But it is clear as the $f_{i}, f_{j}$ commute.

\subsection{Type $\left(B_{2}, 1,2\right)$}

Let $\lambda=2 R \omega_{1}+R^{\prime} \omega_{2} \in P^{\prime}$. Let $\Lambda=\{(i, 1) \mid 1 \leq i \leq 2 R\} \cup\{(i, j) \mid 2 R+1 \leq i \leq 2 R+$ $\left.R^{\prime}, 1 \leq j \leq 2\right\}$. Then $\mathcal{B}(\lambda)$ is isomorphic [22] to the crystal of tableaux $\left(T_{i, j}\right)_{(i, j) \in \Lambda}$ with coefficients in $\{1,2, \overline{2}, \overline{1}\}$ which are semi-standard (i.e., $T_{i, j} \preceq T_{i+1, j}$ and any $i, j$, and $T_{i, 1} \succ T_{i, 2}$ for $i \geq 2 R+1$ for the ordering $1 \preceq 2 \preceq \overline{2} \preceq \overline{1}$ ) and such that for $i \geq 2 R+1,\left(T_{i, 1}, T_{i, 2}\right) \neq(\overline{1}, 1)$ and $\left(T_{i+1,1}, T_{i, 2}\right) \neq(\overline{2}, 2)$.

Let $T_{\lambda}$ be the highest weight tableaux. The tableaux $T=T_{\epsilon}(a, b, c, d)$ is characterized by $a, b, c, d$ and $\epsilon \in\{0,1\}$ such that

$$
\begin{aligned}
T_{i, 1}= \begin{cases}1 & \text { for } i \leq a-1, \\
2 & \text { for } a \leq i \leq b-1, \\
\overline{2} & \text { for } b \leq i \leq c-1, \\
\overline{1} & \text { for } c \leq i,\end{cases} \\
\text { and } T_{i, 2}= \begin{cases}1 & \text { for } 2 R+1 \leq i \leq c-\epsilon-1, \\
2 & \text { for } c-\epsilon \geq i \leq d-1, \\
\overline{2} & \text { for } d \leq i .\end{cases}
\end{aligned}
$$

In fact, $\left(\frac{2}{2}\right)$ appears at most once (it can appear in $T_{1}$ and does not appear in $T_{0}$ ). 
Let us compute the tableaux $T$ of highest weight for the operators $e_{1}^{L}=e_{1}^{2}$ and $e_{2}^{L}=e_{2}$. The condition $e_{2} T=0$ implies $d=R+R^{\prime}+1$. The condition $e_{1}^{2} T=0$ implies $c=d=R+R^{\prime}+1$. Let us consider the 3 classes of such tableaux:

Tableaux (A): $T_{R, 1}=\overline{1}$ (that is $c \leq R$ ). $e_{2} T=0$ gives $R^{\prime} \geq c-b \cdot e_{1}^{2} T=0$ gives $R^{\prime}=0=c-b$ and $2 R \leq a$. So all coefficients are equal to 1 except $T_{2 R, 1} \in\{1,2, \overline{1}\}$.

Tableaux (B): $T_{R, 1}=\overline{\overline{2}}$ (that is $c>R$ and $b \leq R$ ). $e_{2} T=0$ gives $b=R+R^{\prime}+1-\epsilon$. So $\epsilon=1$ and $R^{\prime}=1$. $e_{1}^{2} T=0$ gives $a \geq 2 R$. So $\left(T_{R+1,1}, T_{R+1,2}\right)=(\overline{2}, 2)$, $T_{R, 1} \in\{1,2\}$ and all other coefficients are equal to 1 .

Tableaux (C): $T_{1,1} \preceq 2$ (that is $b>R$ ). $e_{2} T=0$ gives $b=R+R^{\prime}+1-\epsilon$. Then $e_{1}^{2} T=0$ gives $a \geq 2 R$.

For $R^{\prime}=0$ and $R>0$ : we get 3 tableaux $T_{\lambda}, f_{1} T_{\lambda}, f_{1} f_{2} f_{1} T_{\lambda}$.

For $R=0$ and $R^{\prime}>0$ : we get 2 tableaux $T_{\lambda}, f_{1} f_{2} f_{1} T_{\lambda}$.

For $R, R^{\prime}>0$ : we get 4 tableaux

$$
\begin{aligned}
& T_{\lambda}=\left(\begin{array}{llllll} 
& & 1 & \cdots & 1 \\
1 & \cdots & 1 & 2 & \cdots & 2
\end{array}\right), \\
& T_{1}=f_{1} T_{\lambda}=\left(\begin{array}{llllllll}
1 & \ldots & 1 & 2 & 2 & \cdots & 2
\end{array}\right), \\
& T_{2}=f_{1} f_{2} T_{\lambda}=\left(\begin{array}{llllllll}
1 & \cdots & 1 & 2 & \cdots & 2 & 2
\end{array}\right), \\
& T_{3}=f_{1} f_{2} f_{1} T_{\lambda}=\left(\begin{array}{llllllll}
1 & & & & & & & \\
1 & \cdots & 1 & 2 & 2 & \cdots & 2 & 2
\end{array}\right) .
\end{aligned}
$$

We concentrate on the case $R, R^{\prime}>0$ (the cases $R=0$ or $R^{\prime}=0$ can be easily deduced from it). By Theorem 2 the connected component of $T_{\lambda}$ is isomorphic to the crystal of a simple ${ }^{L} \mathfrak{g}$-module. In particular it contains $T_{1}, T_{2}, T_{3}$. Let $\mathcal{B}$ (resp. $\mathcal{B}^{\prime}$ ) be the union of the component of $T_{1}, T_{2}$ (resp. the component of $T_{3}$ ). We have $u \in \mathcal{B}$ if and only if $\operatorname{wt}(u) \in \lambda-(1+2 \mathbb{Z}) \alpha_{1}-\mathbb{Z} \alpha_{2}$. So the component $\mathcal{B} \cap \mathcal{B}^{\prime}=\emptyset$. In the monomial model $\mathcal{M}\left(Y_{2,0}^{R^{\prime}} Y_{1,1}^{2 R}\right), T_{3}$ corresponds to $Y_{1,1}\left(Y_{2,0}^{R^{\prime}} Y_{1,1}^{2(R-1)}\right) Y_{1,5}^{-1}$. By Theorem 4 the ${ }^{L} \mathfrak{g}$-crystal generated by $Y_{2,0}^{R^{\prime}} Y_{1,1}^{2(R-1)}$ is the crystal of the simple ${ }^{L} \mathfrak{g}$-module of highest weight $(R-1) \check{\omega}_{1}+R^{\prime} \check{\omega}_{2}$. But the multiplication by $Y_{1,1} Y_{1,5}^{-1}$ does not change the action of the crystal operators here, and so $\mathcal{B}_{3}$ is also isomorphic to this crystal.

For $\mathcal{B}$ we write explicitly the bijection by using the three cases as above. To do it we also use the dual tableaux realization of $\mathcal{B}^{L}(\mu)$ for $\mu=\mu_{1} \check{\omega}_{1}+\mu_{2} \check{\omega}_{2}$.

Let $\Lambda^{L}=\left\{(i, 2) \mid \mu_{1}<i \leq \mu_{1}+\mu_{2}\right\} \cup\left\{(i, j) \mid 1 \leq i \leq \mu_{1}, 1 \leq j \leq 2\right\}$. $\mathcal{B}^{L}(\mu)$ is isomorphic [22] to the crystal of tableaux $\left(T_{i, j}\right)_{(i, j) \in \Lambda^{L}}$ with coefficients in $\{1,2, \overline{2}, \overline{1}\}$ which are semi-standard as above. The tableaux $T=T_{\epsilon}^{l}(a, b, c, d)$ is characterized by $a, b, c, d$ and $\epsilon \in\{0,1\}$ such that

$$
T_{i, 1}=\left\{\begin{array}{ll}
2 & \text { for } i \leq a-1, \\
\overline{2} & \text { for } a \leq i \leq b-1, \\
\overline{1} & \text { for } b \leq i \leq \mu_{1},
\end{array} \quad \text { and } \quad T_{i, 2}= \begin{cases}1 & \text { for } i \leq b-\epsilon-1, \\
2 & \text { for } b-\epsilon \geq i \leq c-1 \\
\overline{2} & \text { for } c \leq i \leq d-1, \\
\overline{1} & \text { for } d \leq i\end{cases}\right.
$$


Let $\mathcal{B}_{1}^{L}=\mathcal{B}^{L}\left(R \check{\omega}_{1}+\left(R^{\prime}-1\right) \check{\omega}_{2}\right)$ and $\mathcal{B}_{2}^{L}=\mathcal{B}^{L}\left((R-1) \check{\omega}_{1}+\left(R^{\prime}+1\right) \check{\omega}_{2}\right)$. We define $\Psi: \mathcal{B}_{1}^{L} \sqcup \mathcal{B}_{2}^{L} \rightarrow \mathcal{B}$. The general idea to define the map is to replace $(1,1)$, $(2,2),(2, \overline{2}),(\overline{2}, \overline{2}),(\overline{1}, \overline{1})$ in the first part of the tableaux, respectively, by $\left(\begin{array}{l}1 \\ 2\end{array}\right),\left(\frac{1}{2}\right)$, $\left(\frac{2}{2}\right),\left(\frac{2}{1}\right),\left(\frac{2}{1}\right)$, and to replace $\left(\begin{array}{l}1 \\ 2\end{array}\right),\left(\frac{1}{2}\right),\left(\frac{2}{2}\right),\left(\frac{2}{1}\right),\left(\frac{2}{1}\right)$ in the second part of the tableaux, respectively, by $(1),(2),(),(\overline{2}),(\overline{1})$. In general, it cannot be done in the obvious way as other term may appear as $(1,2),(2, \overline{1})$ and so we have to do the following case by case description.

Tableaux (C). $T_{ \pm}(a, b, c, d) \in \mathcal{B} \Leftrightarrow a \equiv \epsilon[2]$. Let $\beta \geq R+1$. We set :

$$
\begin{aligned}
& T_{0}(\alpha, \beta, \gamma, \delta) \in \mathcal{B}_{1}^{L} \mapsto T_{1}(2 \alpha-1, \beta+R, 1+R+\gamma, 1+R+\delta), \\
& T_{0}(\alpha, \beta, \gamma, \delta) \in \mathcal{B}_{2}^{L} \mapsto T_{0}(2 \alpha, \beta+R, R+\gamma, R+\delta) .
\end{aligned}
$$

Tableaux (B). $T_{ \pm}(a, b, c, d) \in \mathcal{B} \Leftrightarrow a \equiv \epsilon[2]$. Let $\beta \leq R<\gamma$. We set

$$
\begin{aligned}
& T_{\epsilon}(\alpha, \beta, \gamma, \delta) \in \mathcal{B}_{1}^{L} \mapsto T_{1}(2 \alpha-1,2 \beta-1-\epsilon, 1+R+\gamma, 1+R+\delta), \\
& T_{\epsilon}(\alpha, \beta, \gamma, \delta) \in \mathcal{B}_{2}^{L} \mapsto T_{0}(2 \alpha, 2 \beta-\epsilon, R+\gamma, R+\delta) .
\end{aligned}
$$

Tableaux (A). $T_{0}(a, b, c, d) \in \mathcal{B} \Leftrightarrow c \equiv a+1[2]$. Let $\gamma \leq R$. We set

$$
T_{\epsilon}(\alpha, \beta, \gamma, \delta) \in \mathcal{B}_{1}^{L} \mapsto T_{0}(2 \alpha-1,2 \beta-\epsilon-1,2 \gamma, 1+R+\delta),
$$

and the image of $T_{\epsilon}(\alpha, \beta, \gamma, \delta) \in \mathcal{B}_{2}^{L}$ is set to be

$$
\begin{cases}T_{0}(2 \alpha, 2 \beta-\epsilon, 2 \gamma-1, R+\delta) & \text { if }(\epsilon=1 \text { or } \beta<\gamma) \text { and } \delta>R, \\ T_{0}(2 \alpha-1,2 \beta, 2 \beta, R+\delta) & \text { if } \epsilon=0, \beta=\gamma \text { and } \delta>R, \\ T_{0}(2 \alpha-1,2 \beta-1-\epsilon, 2 \gamma, 2 R+1) & \text { if } \delta=R .\end{cases}
$$

It is straight forward to check that the properties of Conjecture 3 are satisfied.

Remark 8 In the course of the proof we have found the following Langlands duality branching rules for irreducible representations of $\left(B_{2}, 2,1\right):\left(\lambda_{1}, \lambda_{2}>0\right)$

$$
\begin{aligned}
\Pi\left(\chi\left(2 \lambda_{1} \omega_{1}+\lambda_{2} \omega_{2}\right)\right)= & \chi^{L}\left(\lambda_{1} \check{\omega}_{1}+\lambda_{2} \check{\omega}_{2}\right)+\chi^{L}\left(\lambda_{1} \check{\omega}_{1}+\left(\lambda_{2}-1\right) \check{\omega}_{2}\right) \\
& +\chi^{L}\left(\left(\lambda_{1}-1\right) \check{\omega}_{1}+\left(\lambda_{2}+1\right) \check{\omega}_{2}\right)+\chi^{L}\left(\left(\lambda_{1}-1\right) \check{\omega}_{1}+\lambda_{2} \check{\omega}_{2}\right), \\
\Pi\left(\chi\left(2 \lambda_{1} \omega_{1}\right)\right)= & \chi^{L}\left(\lambda_{1} \check{\omega}_{1}\right)+\chi^{L}\left(\left(\lambda_{1}-1\right) \check{\omega}_{1}+\check{\omega}_{2}\right)+\chi^{L}\left(\left(\lambda_{1}-1\right) \check{\omega}_{1}\right), \\
\Pi\left(\chi\left(\lambda_{2} \omega_{2}\right)\right)= & \chi^{L}\left(\lambda_{2} \check{\omega}_{2}\right)+\chi^{L}\left(\left(\lambda_{2}-1\right) \check{\omega}_{2}\right) .
\end{aligned}
$$

\subsection{A proposed deformation process}

Suppose that $r=2$. We have proved the statement of Conjecture 3 for rank 2 , but we cannot use Theorem 5 directly for general rank. For example, for type $B_{3}, \tilde{\mathcal{B}}(\lambda)$ is a 
normal crystal for ${ }^{L} \mathfrak{g}_{\{1,2\}}$ and ${ }^{L} \mathfrak{g}_{\{1,3\}}$. We use the rank 2 to deform the 3 -arrows so that we get ${ }^{L} \mathfrak{g}_{\{2,3\}}$. But then we may not preserve the ${ }^{L} \mathfrak{g}_{\{1,3\}}$-crystal structure.

We propose a conjectural inductive process to redefine the crystal operators of $\tilde{\mathcal{B}}(\lambda)$ so that we get a normal crystal. Suppose that we know the result for rank lower than $n-1$ for an $n \geq 3$. Let $I=I_{1} \sqcup I_{2}$ where $I_{k}=\left\{i \in I \mid r_{i}=k\right\}$. We assume $\left|I_{2}\right| \geq 2$ (the case $\left|I_{1}\right| \geq 2$ can be treated in a symmetric way by Proposition 8$)$. We use the notation $\underline{I_{2}}=\left\{1, \ldots, i_{0}\right\}$ and $I_{1}=\left\{i_{0}+1, \ldots, n\right\}$ so that $C_{i_{0}, i_{0}+1}=-1$. Let $\overline{I_{2}}=I_{2}-\left\{i_{0}\right\}$, $\overline{I_{1}}=I_{1} \cup\left\{i_{0}\right\}$.

Let $\lambda \in P^{\prime}$ and fix a class $P^{\prime \prime}=\mu+Q^{\prime \prime} \subset \lambda+Q \bmod Q^{\prime}$. Then $\mathcal{B}=\{u \in$ $\left.\tilde{\mathcal{B}}(\lambda) \mid \operatorname{wt}(u) \in P^{\prime \prime}\right\}$ is a union of connected component of $\tilde{\mathcal{B}}(\lambda)$ as the weight of the vectors in a connected component are in the same class. For $\mu_{1}, \mu_{2} \in P^{\prime \prime}$, we have $\mu_{1}-\mu_{2}=\sum_{i \in I} n_{i} \alpha_{i}^{L}$ where $n_{i} \in \mathbb{Z}$ and the $\alpha_{i}^{L}$ are the simple roots of ${ }^{L} \mathfrak{g}$. We put $N\left(\mu_{1}, \mu_{2}\right)=\sum_{i \in I} n_{i}$. Let $\mu^{\prime} \in\{\operatorname{wt}(u) \mid u \in \mathcal{B}\}$ such that $N\left(\mu, \mu^{\prime}\right)$ is maximal. It is well defined, that is to say independent of the choice of $\mu$, as for $\mu_{1}, \mu_{2}, \mu_{3} \in P^{\prime \prime}$ we have $N\left(\mu_{1}, \mu_{2}\right)+N\left(\mu_{2}, \mu_{3}\right)=N\left(\mu_{1}, \mu_{3}\right)$. We set $N\left(\mu_{1}\right)=N\left(\mu^{\prime}, \mu_{1}\right)$. For $N \geq 0$, let $W_{N}=\{u \in \mathcal{B} \mid N(\operatorname{wt}(u))=N\}$.

For $\mathcal{C}$ a (normal) ${ }^{L} \mathfrak{g}$ crystal, by truncated (normal) crystal of $\mathcal{C}$ we mean for a certain $N \in \mathbb{Z}$ the set $\{u \in \mathcal{C} \mid N(\operatorname{wt}(u)) \geq N\}$ with the maps wt ${ }^{L}, e_{i}^{L}, \epsilon_{i}, \phi_{i}$ restricted to it and the map $f_{i}^{L}$ restricted to $\{u \in \mathcal{C} \mid N(\operatorname{wt}(u)) \geq N-1\}$.

To start we set all $\left(f_{i}^{L}\right)^{\prime}=f_{i}^{L},\left(e_{i}^{L}\right)^{\prime}=e_{i}^{L}$. By induction on $N \geq 0$, we redefine $\left(f_{i}^{L}\right)^{\prime}$ on $\sqcup_{M \leq N-1} W_{M}$ (or equivalently $\left(e_{i}^{L}\right)^{\prime}$ on $\left.\sqcup_{M \leq N} W_{M}\right)$. We say that the process does not fail if $\left(\sqcup_{M \leq N} W_{M}\right.$, wt $\left.{ }^{L}, \epsilon_{i}^{L}, \phi_{i}^{L},\left(f_{i}^{L}\right)^{\prime},\left(e_{i}^{L}\right)^{\prime}\right)$ is a normal truncated crystal.

For $N=0$ we do not change the maps. Let $N \geq 0$.

Let $i \in I_{1}$ and $u \in W_{N-1}$ such that $\exists j \in \bar{I}_{2}, \epsilon_{j}^{L}(u)>0$. If $\phi_{i}^{L}(u)=0$ we set $\left(f_{i}^{L}\right)^{\prime}(u)=f_{i}^{L}(u)=0$. Otherwise let $v=e_{j}^{L}(u) \neq 0$. Then $\phi_{i}^{L}(v)=\phi_{i}^{L}(u) \neq 0$ so $w=\left(f_{i}^{L}\right)^{\prime}(v) \neq 0$. Then $\phi_{j}^{L}(w)=\phi_{j}^{L}(v) \neq 0$ so $x=f_{j}^{L}(w) \neq 0$. We set $\left(f_{i}^{L}\right)^{\prime}(u)=x$.

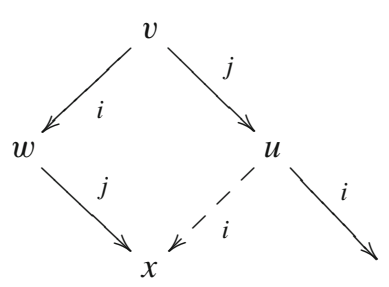

We have $\epsilon_{i}^{L}(x)=\epsilon_{i}^{L}(w)=\epsilon_{i}^{L}(v)+1=\epsilon_{i}^{L}(u)+1$ and $\left(f_{i}^{L}\right)^{\prime}(u)$ is well-defined (independent on $\left.j \in \overline{I_{2}}\right)$. $\left(e_{i}^{L}\right)^{\prime}(y)$ is now defined for $y \in W_{N}$ such that $\sum_{j \in \bar{I}_{2}} \epsilon_{j}^{L}(u)>0$.

Let $\mu \in P^{\prime}$ and $\mathcal{U}_{ \pm}=\left\{y \in\left(W_{N}\right)_{\mu} \mid \pm \sum_{j \in \bar{I}_{2}} \epsilon_{j}^{L}(y) \leq \pm 1 / 2\right\}$. We redefine $\left(e_{i}^{L}\right)^{\prime}$ on $\mathcal{U}_{+}$by induction on $i \geq i_{0}$. Let

$$
u \in \mathcal{B}_{i}=\left\{u \in\left(W_{N}\right)_{\mu+\alpha_{i}} \mid \phi_{i}^{L}(u)>0, u \notin\left(e_{i}^{L}\right)^{\prime}\left(\mathcal{U}_{-}\right)\right\} .
$$

Consider the truncated ${ }^{L} \mathfrak{g}$-crystal $\sqcup_{M \leq N-1} W_{M}$ and $\mathcal{C}$ be the corresponding normal crystal with the injection $\Psi: \sqcup_{M \leq N-1} W_{M} \rightarrow \mathcal{C}$. We have $\phi_{i}^{L}(\Psi(u))=\phi_{i}^{L}(u)>0$ 
and so $v=f_{i}^{L}(\Psi(u)) \neq 0$. If there is $i_{0} \leq j \leq i-1$ such that $\epsilon_{j}^{L}(v)>0$, let $w=e_{j}^{L}(v)$. We set $\left(f_{i}^{L}\right)^{\prime}(u)=\left(f_{j}^{L}\right)^{\prime} \Psi^{-1}(w)=x$.
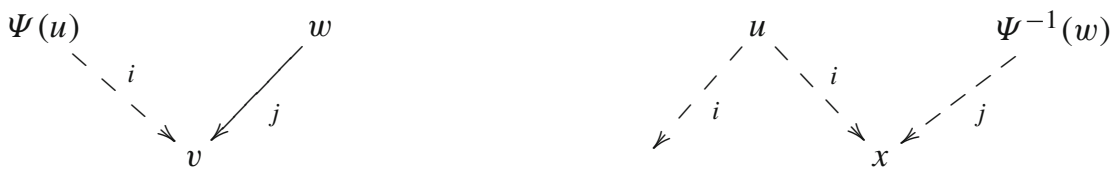

As $\left|\overline{I_{1}}\right|<n$ we have :

$$
\begin{gathered}
\left|\left\{v \in \mathcal{B}_{i} \mid\left(\epsilon_{j}^{L}\left(f_{i}^{L}(\Psi(u))\right)\right)_{j \in \overline{I_{1}}}=\left(a_{j}\right)_{j \in \overline{I_{1}}}\right\}\right| \\
=\left|\left\{v \in \mathcal{U}_{+} \mid\left(\epsilon_{j}^{L}(v)\right)_{j \in \overline{I_{1}}}=\left(a_{j}\right)_{j \in \overline{I_{1}}}\right\}\right|
\end{gathered}
$$

for a given $\left(a_{j}\right)_{j \in \overline{I_{1}}}$. So we can define $\left(f_{i}^{L}\right)^{\prime}(u)$ for $u \in \mathcal{B}_{i}$ such that $\sum_{i_{0} \leq j \leq i-1} \epsilon_{j}(v)=$ 0 . We get $\left(f_{i}^{L}\right)^{\prime}: \mathcal{B}_{i_{0}} \rightarrow \mathcal{U}_{+}$injection. Moreover conjecturally for $i=i_{0}$, we can choose $\left(f_{i_{0}}^{L}\right)^{\prime}$ compatible with the $\phi_{i}^{L}, i \in \overline{I_{2}}$ (in other words, there is "enough dimension" in weight spaces to do it) and then we can redefine $\left(f_{i_{0}}^{L}\right)^{\prime}$ on $\mathcal{U}_{+}$so that the structure of ${ }^{L} \mathfrak{g}_{I_{2}}$-crystal is not modified.

If the conjectural point is satisfied, the process never fails, and the new crystal is normal for any ${ }^{L} \mathfrak{g}_{J}$ where $|J| \leq 2$. Then we can conclude with Theorem 5 .

Acknowledgments This work was begun while we were taking part in the Program on Combinatorial Representation Theory held at MSRI in the Spring of 2008. We thank the organizers of this Program for their invitations and MSRI for hospitality.

Open Access This article is distributed under the terms of the Creative Commons Attribution Noncommercial License which permits any noncommercial use, distribution, and reproduction in any medium, provided the original author(s) and source are credited.

\section{References}

1. Beilinson, A., Drinfeld, V.: Quantization of Hitchin's integrable system and Hecke eigensheaves. http://www.math.uchicago.edu/ mitya/langlands.html

2. Benkart, G., Witherspoon, S.: Restricted two-parameter quantum groups. In: Fields Institute Communications, "Representations of Finite Dimensional Algebras and Related Topics in Lie Theory and Geometry", vol. 40, pp. 293-318. American Mathematical Society, Providence (2004)

3. Chari, V., Pressley, A.: A Guide to Quantum Groups. Cambridge University Press, Cambridge (1994)

4. Feigin, B., Frenkel, E.: Affine Kac-Moody algebras at the critical level and Gelfand-Dikii algebras. In: Tsuchiya, A., Eguchi, T., Jimbo, M. (eds.) Infinite Analysis. Adv. Ser. in Math. Phys., vol. 16, pp. 197-215. World Scientific, Singapore (1992)

5. Frenkel, E.: Wakimoto modules, opers and the center at the critical level. Adv. Math. 195, 297-404 (2005)

6. Frenkel, E.: Lectures on the Langlands program and conformal field theory. In: Cartier, P., et al. (eds.) Frontiers in Number Theory, Physics and Geometry II, pp. 387-536. Springer, Berlin (2007). hep-th/0512172

7. Frenkel, E.: Langlands Correspondence for Loop Groups. Cambridge Studies in Advanced Mathematics, vol. 103. Cambridge University Press, Cambridge (2007) 
8. Frenkel, E., Gaitsgory, D.: Affine Kac-Moody algebras and local geometric Langlands correspondence (with D. Gaitsgory). In: Algebraic Geometry and Number Theory. Progress in Math., vol. 253, pp. 69-260. Birkhäuser, Boston (2006). math.RT/0508382

9. Frenkel, E., Hernandez, D.: Langlands duality for finite-dimensional representations of quantum affine algebras. Preprint. arXiv:0902.0447

10. Frenkel, E., Reshetikhin, N.: Quantum affine algebras and deformations of the Virasoro and $W$-algebras. Commun. Math. Phys. 178(1), 237-264 (1996)

11. Frenkel, E., Reshetikhin, N.: Deformations of $W$-algebras associated to simple Lie algebras. Commun. Math. Phys. 197(1), 1-32 (1998)

12. Frenkel, E., Reshetikhin, N.: The $q$-characters of representations of quantum affine algebras and deformations of $W$-algebras. In: Recent Developments in Quantum Affine Algebras and Related Topics (Raleigh, NC, 1998). Contemp. Math., vol. 248, pp. 163-205. American Mathematical Society, Providence (1999)

13. Gelfand, I.M., Kirillov, A.A.: The structure of the Lie skew field related to a split semi-simple Lie algebra. Funct. Anal. Appl. 3(1), 6-21 (1969)

14. Hernandez, D.: The Kirillov-Reshetikhin conjecture and solutions of $T$-systems. J. Reine Angew. Math. 596, 63-87 (2006)

15. Hernandez, D.: Kirillov-Reshetikhin conjecture: the general case. Int. Math. Res. Not. IMRN 2010(1), 149-193 (2009)

16. Hernandez, D., Nakajima, H.: Level 0 monomial crystal, Lusztig's issue. Nagoya Math. J. 184, 85-153 (2006)

17. Kac, V.: Lie superalgebras. Adv. Math. 26(1), 8-96 (1977)

18. Kashiwara, M.: Similarity of Crystal Bases, Lie Algebras and Their Representations (Seoul, 1995). Contemp. Math., vol. 194. pp. 177-186. American Mathematical Society, Providence (1996)

19. Kashiwara, M.: Realizations of crystals, in Combinatorial and geometric representation theory (Seoul, 2001). Contemp. Math., vol. 325. pp. 133-139. American Mathematical Society, Providence (2003)

20. Kashiwara, M.: Bases cristallines des groupes quantiques. Charles Cochet (ed.) Cours Spécialisés, vol. 9. Société Mathématique de France, Paris (2002)

21. Kashiwara, M., Kang, S.-J., Misra, K., Miwa, T., Nakashima, T., Nakayashiki, A.: Affine crystals and vertex models. Int. J. Mod. Phys. A 7(Suppl 1A), 449-484 (1992) (Proceeding of the RIMS Research Project 1991 Infinite Analysis)

22. Kashiwara, M., Nakashima, T.: Crystal graphs for representations of the $q$-analogue of classical Lie algebras. J. Algebra 165, 295-345 (1994)

23. McGerty, K.: Langlands duality for representations and quantum groups at a root of unity. Commun. Math. Phys. 296(1), 89-109 (2010)

24. Nakajima, H.: $t$-Analogs of $q$-characters of quantum affine algebras of type $A_{n}, D_{n}$. In: Combinatorial and Geometric Representation Theory (Seoul, 2001). Contemp. Math., vol. 325, pp. 141-160. American Mathematical Society, Providence (2003)

25. Reshetikhin, N.: Multiparameter quantum groups and twisted quasitriangular Hopf algebras. Lett. Math. Phys. 20, 331-335 (1990) 